\title{
Linx
}

Revue des linguistes de l'université Paris X Nanterre

$54 \mid 2006$

La cause : approche pluridisciplinaire

\section{Les sémantèmes de causation en français}

\section{Sylvain Kahane et Igor Mel'čuk}

\section{OpenEdition}

\section{Journals}

Édition électronique

URL : http://journals.openedition.org/linx/539

DOI : $10.4000 /$ linx.539

ISSN : 2118-9692

\section{Éditeur}

Presses universitaires de Paris Nanterre

\section{Édition imprimée}

Date de publication : 1 juin 2006

Pagination : 247-292

ISSN : 0246-8743

\section{Référence électronique}

Sylvain Kahane et Igor Mel'čuk, "Les sémantèmes de causation en français », Linx [En ligne], 54 | 2006, mis en ligne le 01 août 2007, consulté le 19 avril 2019. URL : http:// journals.openedition.org/linx/539; DOI : 10.4000/linx.539 


\title{
Les sémantèmes de causation en français
}

\author{
Sylvain Kabane E Igor Mel'čuk \\ Modyco, Université Paris 10 - OLST, Université de Montréal
}

\section{Introduction : le problème posé}

Le but de cette étude est strictement et purement linguistique: une description du sens langagier 'causer', ou, plus précisément, du ou des sens du verbe CAUSER en français, ainsi que des verbes exprimant une causation. Pour être bien compris, nous nous devons, dans un premier temps, de nous démarquer des études physico-philosophiques et logico-psychologiques du concept de causation.

- Les études physico-philosophiques de la causation (en physique, en théorie des sciences, en philosophie, etc.) posent la question sur la causation de la façon suivante :

Quelles conditions caractérisant une situation du monde réel doivent être présentes pour qu'on ait le droit d'y appliquer le concept de causation?

Les recherches de ce type sont bien illustrées par la définition suivante de la causation (Kistler 1999 : 282) :

Deux événements $\mathbf{c}$ et $\mathbf{e}$ sont liés comme cause et effet si et seulement s'il existe au moins une grandeur physique $\mathrm{P}$, soumise à une loi de conservation, exemplifiée dans $\mathbf{c}$ et $\mathbf{e}$, et dont une quantité déterminée est transférée entre $\mathbf{c}$ et $\mathbf{e}$.

- Les études logico-psychologiques (en logique, en psychologie, dans les sciences cognitives, etc.) s'intéressent à une autre question : 
Sylvain Kahane \& Igor Mel'cuk

Quelles conditions caractérisant les croyances d'une personne et ses connaissances du monde réel doivent être présentes pour qu'elle puisse raisonner en se basant sur le concept de causation?

Pour les recherches de ce type, nous pouvons citer Goldvarg \& Johnsson-Laird 2001 :

[Notre] théorie décrit comment les gens raisonnent sur la causalité et essaie de résoudre les trois casse-têtes suivants : qu'est-ce que signifient les relations causales, comment sont-elles représentées mentalement et comment les gens font-ils des inférences à partir d'elles » [c'est nous qui traduisons. - SK, IM].

Dans le cadre physico-philosophique ou logico-psychologique, on cherche donc à modéliser certaines propriétés intrinsèques de l'univers ou de la psyché, tout en laissant de côté les liens de ces propriétés avec les langues naturelles. Les concepts étudiés ne correspondent pas directement au sens de mots d'une langue particulière et doivent être les mêmes que l'étude soit en français, en russe ou en japonais. Le choix de termes tels que causation, lien causal ou relation de cause à effet n'est pas bien entendu fortuit, mais les études physiques ou psychologiques ne sont pas du tout centrées sur le sens de ces mots en français ou de leurs équivalents dans d'autres langues. La littérature physico-philosophique ou logico-psychologique consacrée à la causation est énorme ; nous nous limiterons à mentionner quelques ouvrages où le lecteur intéressé pourra trouver une riche bibliographie ainsi que des tours d'horizon appropriés (Tooley 1987, 1997, Ehring 1997, Goldvarg \& Johnson-Laird 2001).

La tâche que nous nous donnons dans cet article est différente : elle est, comme nous venons de le dire, purement linguistique. Nombre de chercheurs ont traité, depuis une trentaine d'années, des aspects linguistiques de la causation: Shibatani 1973, Comrie 1974, 1985, Givón 1975, Shibatani (ed.) 1976, Talmy 1988, Apresjan 1998 [1976], Song 1996, Paducheva 1997, pour n’indiquer que quelques-uns des ouvrages les plus influents. Parmi ceux-ci, retenons les articles assez récents de Wierzbicka 1998, 2004, où différentes acceptions des verbes de causation en anglais sont distinguées et sémantiquement décomposées; nous retournerons à Wierzbicka 1998 dans la section 5. Quant aux études linguistiques visant spécifiquement la causation en français, les recherches sont également nombreuses; par exemple, une discussion de la causation en français est offerte dans St-Germain 1995 ; la notion de cause dans la langue appliquée à quelques connecteurs discursifs français est analysée dans Anscombre 1984 ; plusieurs conjonctions et prépositions causales du français sont étudiées dans Iordanskaja 1999 et Iordanskaja \& Arbatchewski-Jumarie 2000, ainsi que Nazarenko 2000, qui contient une bibliographie importante; Gross 2005 ébauche un projet de longue haleine consacré aux différentes expressions de la causation en français. Faute d'espace, nous ne pouvons pas analyser ici les résultats pertinents de ces recherches; nous allons tout simplement exposer notre approche de la description des sens causatifs dans les langues naturelles.

Du point de vue linguistique, deux questions peuvent être formulées au sujet de la causation. La première, bien que radicalement différente des questions que se posent les physiciens et les philosophes d'une part, les logiciens et les psychologues d'autre part, s'y apparente dans sa formulation : 
1) Quelles conditions caractérisant (une représentation d')une situation du monde réel doivent être présentes pour qu'on ait le droit d'y appliquer le sens langagier 'causer' ?

Il s'agit d'une correspondance entre une représentation conceptuelle d'une situation réelle et une représentation sémantique des phrases d'une langue particulière qui décrivent cette situation. Mais nous n'aborderons pas cette question directement. Nous allons plutôt nous attaquer à la deuxième question :

2) Quelles conditions caractérisant une représentation sémantique d'une phrase française qui inclut le sens 'causer' doivent être présentes pour qu'on ait le droit d'exprimer ce sens en français par tel ou tel moyen linguistique (une unité lexicale ou une construction syntaxique donnée)?

Il s'agit d'une correspondance entre une représentation sémantique des phrases et les phrases elles-mêmes, ou, plus exactement, une représentation formelle de ces phrases.

Nous posons donc que la production langagière se fait en deux étapes majeures: 1) le passage du monde réel au sens langagier et 2) le passage du sens langagier à l'expression langagière ; notre étude sera centrée sur cette deuxième étape.

Le contraste entre une étude physico-philosophique et une étude linguistique de la causation peut être mis en évidence par les exemples suivants. Soient quatre situations réelles de causation comparables du point de vue physique, où un événement qui affecte une grenouille (le Déclencheur) en entraîne un autre (le Résultat) :

\section{Déclencheur}

a. Une valise tombe sur la grenouille

b. Une bombe explose près de la grenouille

c. Zoé tire avec sa carabine une balle sur la grenouille $\Rightarrow$ La grenouille meurt.

d. Un fil électrique envoie une décharge à la grenouille $\Rightarrow$ La grenouille meurt.

Du point de vue linguistique, la description de ces quatre situations en français regorge de divergences. Ainsi, le nom de l'événement déclencheur - chute, explosion, tir, (l'envoi d'une) décharge - peut ou non être pris comme sujet du verbe TUER $(\approx$ 'causer la mort') : (2)a, b vs (2)c, d ; le premier argument sémantique de cet événement - valise, bombe, Zoé, fil électrique - aussi peut ou non être pris comme sujet: (2)a, b, c vs (2)d ; enfin, d'autres arguments de TUER peuvent ou non être pris comme sujet: la balle vs la carabine en (2)c.

$$
\begin{aligned}
& \text { a. La valise a tué la grenonille. } \\
& \text { ? }{ }^{*} \text { a chute de la valise a tué la grenonille } 1 . \\
& \text { La grenonille a été tuée par la chute de la valise. }
\end{aligned}
$$

\footnotetext{
${ }^{1}$ Les jugements d'acceptabilité pour des exemples comme La chute de la valise a tué la grenouille - en (2)a - varient ; certains trouvent des expressions de ce type acceptables. En particulier, une phrase comme La chute du plafond a tué 6 personnes est ressentie comme meilleure par la plupart des locuteurs et même parfaitement acceptable pour plusieurs. Comme on le verra à la lumière de notre
} 
Sylvain Kahane \& Igor Mel’čuk

b. La bombe a tué la grenouille.

L'explosion de la bombe a tué la grenouille.

c. Zoé a tué la grenouille.

'Le tir de Zoé a tué la grenouille.

La grenouille a été tuée par le tir de Zoé.

* La carabine a tué la grenouille ${ }^{2}$.

* La grenonille a été tuée par la carabine.

La balle a tué la grenouille.

d. * Le fil électrique a tué la grenouille.

* La grenouille a été tuée par le fil électrique.

La décharge a tué la grenouille.

Si on considère les expressions de la causation dans des langues différentes, la variété des expressions possibles/impossibles devient encore plus prononcée :

(3) a. russe Svoim roždeniem ètot rebënok dostavil roditeljam mnogo radosti lit.

(Par sa naissance cet enfant a causé une grande joie à ses parents).

vs

b. *Par sa naissance, cet enfant a causé une grande joie à ses parents.

vs

c. La naissance de cet enfant a causé une grande joie à ses parents.

(4) a. angl. The plane crash killed all 135 passengers on board.

vs

b. ? ${ }^{*}$ Le crash de l'avion a tué les 135 passagers à bord.

Enfin, l'usage d'une expression donnée obéit à des contraintes multiples. Ainsi, le verbe causatif anglais KILL peut, pour une même situation, être utilisé au participe présent, mais pas à la forme finie avec un sujet comme PLANE :

(5) a. *The plane that crashed yesterday killed all 135 passengers on board.

vs

b. The plane crashed yesterday, killing all 135 passengers on board.

L'objectif d'une description linguistique est de modéliser l'usage des expressions langagières : bloquer les expressions inacceptables et autoriser les autres. Notre article tente d'atteindre cet objectif pour la causation. Ce que nous décrivons est la causation en tant que sens langagier - c'est-à-dire un élément du signifié des verbes français

description, les contraintes qui jouent ici sont en partie de nature pragmatique et dépendent donc de l'interprétation donnée par le locuteur à la situation dont il est question.

${ }^{2}$ Les phrases de ce type sont possibles s'il s'agit de caractériser un objet - comme, par exemple, une carabine exposée dans un musée : Cette carabine a tué des milliers de bisons. Mais ce n'est plus alors la même situation. 
exprimant une causation; la causation est considérée alors sous l'angle strictement lexico-sémantique. Notre tâche se réduit à la description formalisée du lexique par une modélisation du signifié de chaque lexie. Une lexie est une unité lexicale, c'est-à-dire un mot ou un syntagme figé pris dans une acception bien définie. Le signifié d'une lexie est un sémantème. Dans cet article, nous allons donc nous intéresser aux sémantèmes de causation. Nous nous concentrerons en particulier sur le sens de base du verbe français CAUSER, ce sens faisant partie du sens de milliers de verbes, comme NETTOYER (pris dans l'acception qu'on voit dans Ce savon nettoie bien: 'causer que Y devienne propre'), ÉLIMINER (L'acide élimine la rouille: 'causer que Y disparaisse'), TORTURER (Cet abcès me torture), EXPLIQUER (Cette phrase explique tout), IRRITER (Ce bruit m'irrite), TUER (La flèche l'a tué net), ... Pour être plus exact, le sens 'causer' constitue la composante communicativement dominante ${ }^{3}$ de la décomposition sémantique de tels verbes.

Prolongeant le travail de Iordanskaja \& Mel'čuk 2001 : 116-118 (qui développe les idées de Apresjan 1998 : 276), nous proposons de distinguer deux sémantèmes de causation en français : 'causer1' et 'causer2' (et par conséquent deux lexies dont ces sémantèmes sont des signifiés: CAUSER1 et CAUSER2). Le premier sémantème, 'causer1', est le 'causer' non agentif ( $\approx$ 'être la cause de ...'), qui apparaît dans $(6)$ a, et le second, 'causer2', est le 'causer' agentif ( $\approx$ 'être le causateur de ...'), qu'on trouve dans (6)b :

(6) a. L'acide a dissous ['a causé1 la dissolution de'] le tissu.

b. Zoé a dissous ['a causé2 la dissolution de’] le tissu (avec de l'acide/dans l'acide).

Le résultat en est que pour beaucoup de verbes causatifs du français, nous distinguons deux acceptions lexicographiques : non agentif et agentif ; cette distinction sera justifiée plus loin (la section 3).

Nous pensons que les deux sémantèmes de causation - 'causer1' et 'causer2' ne sont pas des primitives sémantiques. (Ce point de vue diffère de celui de A. Wierzbicka, qui, depuis des années, propose 'because' = 'parce que' comme une de ses cinquante-cinq primitives sémantiques ; par exemple, Wierzbicka 1996: 70-71, 186188.) Nous présenterons donc leur décomposition sous forme de règles; de telles

\footnotetext{
${ }^{3}$ La composante communicativement dominante dans une structure (réseau) sémantique [= SSém] est la composante qui représente la paraphrase minimale de cette SSém: en réduisant une SSém à sa composante dominante, on perd de l'information, mais on ne la déforme pas (Polguère 1990 : 118122, 1992 : 117 ; Mel'čuk 2001 : 31). La composante communicativement dominante est indiquée par un soulignement. Ainsi, dans la configuration sémantique 'le.général retourne à.l'hôtel', chacune des trois composantes sémantiques peut être choisie comme composante communicativement dominante, ce qui donne :

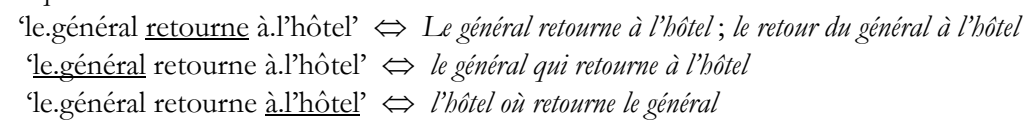

La représentation sémantique d'une phrase elle-même n'a pas en général de nœud communicativement dominant, mais elle est découpée en des aires communicative - entre autres, un thème et un rhème, chacun de ces sous-réseaux ayant son propre nœud dominant (Mel'čuk 2001 : $28 s s v$.).
} 
règles permettent de manipuler des représentations sémantiques en substituant ces sémantèmes à leur décomposition et vice versa.

Pour mieux circonscrire notre tâche, nous renonçons à l'analyse de plusieurs problèmes tout à fait pertinents, surtout les trois suivants :

- l'opposition « causation directe $v$ s causation indirecte » (cf., par exemple, Wolff 2003) ;

- la distinction entre différents types de causation ('causer' vs 'rendre possible' vs 'autoriser' vs '(ne pas) empêcher' ; Goldvarg \& Johnson-Laird 2001) ;

- la question de l'auto-causation (que l'on trouve dans Il lève le bras, Il ferme les yeux, Il court ...; Bogusławski 1991).

La structure du reste de l'article est comme suit : les sections 2 et 3 décrivent, respectivement, les sémantèmes 'causer1'et 'causer2', et la section 4 discute de la décomposition des deux sens de TUER et de celui de angl. HAVE.

\section{Le sémantème 'causer1' (= 'être la cause')}

Le sémantème 'causer1' est un prédicat à trois arguments :

$$
\text { 'causer1'('X', 'Y', ['Z’]), }
$$

qu'on écrira aussi 'X cause1 Y [par Z(X)]'.

L'emploi du sémantème 'causer1' peut être illustré par la phrase 0 , où ce semantème est exprimé par le verbe CAUSER1 :

(7) Le va-et-vient [ ' 'X'] incessant des voitures cause l'irritation [= 'Y'] de Zoé par son bruit $\left[=' \mathrm{Z}(\mathrm{X}){ }^{\prime}\right]$ assourdissant.

En français, le premier argument ' $\mathrm{X}$ ' du sémantème 'causer1' peut être désigné par la lexie CAUSE (de Y) et le deuxième argument 'Y', par la lexie EFFET (de X). Cela nous permet, entre autres, d'assurer les paraphrases suivantes :

(8) Le va-et-vient incessant des voitures cause l'irritation de Zoé.

= Le va-et-vient incessant des voitures est la cause de l'irritation de Zoé.

三L'irritation de Zoé est l'effet du va-et-vient incessant des voitures.

Dans la suite, nous utiliserons les noms Cause et Effet pour désigner les rôles sémantiques remplis par ' $\mathrm{X}$ ' et ' $\mathrm{Y}$ '.

Le troisième argument de 'causer1', 'Z', n'a pas de nom spécial en français ; le rôle sémantique de ' $Z$ ' sera appelé Élaboration (de la Cause).

Les arguments ' $Y$ ' et 'Z' de 'causer1' doivent être des faits, c'est-à-dire que leur décomposition peut être itérée jusqu’à arriver à une structure sémantique dont le sémantème 'fait' est la composante communicativement dominante ${ }^{4}$.

\footnotetext{
${ }^{4}$ Les sémantèmes 'fait' et 'événement', ainsi qu'action'/'agir', jouent un rôle important dans nos descriptions ; voir dans l'Annexe quelques commentaires à leur sujet. Par ailleurs, notre formulation implique que l'on pourra décomposer n'importe quel sémantème référant à un fait jusqu'à ce qu'on tombe sur le sémantème 'fait'. En fait, on tombera avant sur une primitive sémantique, comme, par exemple, 'agirII.1'/'actionII.1', qui, strictement
} 
Quant à 'X', nous n'exigeons pas que ce soit aussi un fait, alors que, dans le traitement habituel de la causation, on part de la prémisse que la causation relie toujours deux faits. C'est vrai si on considère les concepts physique ou logicophilosophique de causation, mais notre sémantème 'causer1' ne représente pas ces concepts : comme nous l'avons déjà dit, nous décrivons ici tout simplement l'usage des verbes français incluant ce sémantème. Nous nous devons donc de rendre compte de leurs particularités de combinatoire que nous croyons liées à la présence de ce sémantème dans leur définition lexicographique. Entre autres, le sémantème français 'causer1', ainsi que n'importe quel verbe dont le sens l'inclut comme composante dominante, permet que son premier argument soit le nom d'une entité physique (Les voitures causent des dégâts importants aux arbres des villes), y compris un individu (Jean me cause des soucis par son aspect maladiff.

Le premier argument ' $\mathrm{X}$ ' de 'causer1' doit en même temps être le premier argument de ' $Z$ ', qui est lui-même un prédicat. L'argument ' $Z$ ' est qualifié d'escamotable, ce qu'indiquent les crochets, parce que la structure sémantique 'causer1'('X', 'Y') - c'est-à-dire sans ' $Z$ ' - est aussi considérée comme bien formée'. Un argument escamotable est toujours « sous-jacent» : dans la structure sémantique de la phrase La poutre a écrasé la voiture, on n'est pas obligé de mentionner l'action ' $Z$ ' de la poutre sur la voiture, mais cette action est nécessairement présente dans la réalité et peut toujours être incluse dans la structure sémantique de départ et exprimée : La poutre a écrasé la voiture en lui tombant dessus ${ }^{6}$. Un argument escamotable est un argument sémantique qui correspond à un participant obligatoire de la situation en question, mais qu'on peut ne pas exprimer dans la structure sémantique. Dans le cas du sémantème 'causer1', ceci est possible grâce à une propriété particulière de ce sémantème que nous allons considérer tout de suite.

Les arguments ' $\mathrm{X}$ ' et ' $\mathrm{Z}$ ' du sémantème 'causer1' manifestent un lien important :

- D’une part, ' $Z$ ' est nécessairement une partie, une propriété, un état, une action, etc. de 'X'. Il s'agit de la relation appelée en linguistique (de façon un peu

parlant, n'est pas décomposable - c'est-à-dire ne peut pas être mis en correspondance avec une configuration de sémantèmes qui lui soit équivalente. Une telle primitive peut néanmoins contenir un autre sémantème, en l'occurrence 'fait'; cela signifie que notre primitive sémantique peut être réduite à 'fait' plus un «morceau» de sens qu'on ne peut pas réduire à une configuration de sémantèmes. De tels résidus irréductibles peuvent être rapprochés des « quarks » sémantiques d'Apresjan (1995: 481-482) : un quark sémantique est un élément sémantique que l'on peut extraire d'une comparaison de sens apparentés comme partie commune, mais pour laquelle il n'existe pas d'expression standard dans la langue (bien que parfois quelques expressions linguistiques très contraintes soient possibles).

${ }^{5}$ Un argument escamotable est différent d'un argument optionnel, tel que 'récipient' pour 'boire (dans un récipient)' : 'récipient' est un argument optionnel de 'boire', puisque la situation'X boit $\mathrm{Y}$ ' est parfaitement envisageable sans un récipient (pour arguments optionnels de prédicats sémantiques, voir Mel'čuk 2004a : 30ssv., 50, où les arguments sont appelés actants sémantiques).

${ }^{6}$ On remarquera que si 'tomber' monovalent (X tombe) n'est pas une action, 'tomber sur qqch.' bivalent ( $X$ tombe sur $Y$ ) implique une action de $\mathrm{X}$ sur $\mathrm{Y}$. Voir la description du sémantème 'actionI.1' plus loin. 
abusive) la relation possessive : ' $Z$ ' est quelque chose de ' $\mathrm{X}$ '. Nous notons cela ' $\mathrm{Z}$ '(' $\mathrm{X}$ '), ce qui, techniquement parlant, signifie que ' $\mathrm{X}$ ' est le premier argument du prédicat ' $Z$ '.

- D'autre part, comme il est courant dans les langues naturelles, ' $\mathrm{X}$ ' et ' $\mathrm{Z}$ ' - le "possédé» et le "possesseur»- peuvent se remplacer mutuellement par une métonymie tout aussi naturelle que répandue (La taille de Zoé est mince $\approx$ Zoé est mince (de taille), La voiture de Zoé est mal garée $\approx$ Zoé est mal garée, etc.). Avec 'causer1', ce processus est récursif :

(9) a. Les voitures causent l'irritation de Zoé (par leur va-et-vient incessant).

b. Le va-et-vient incessant des voitures cause l'irritation de Zoé (par son bruit assourdissant).

c. Le bruit assourdissant du va-et-vient incessant des voitures cause l'irritation de Zoé (par son impact sur ses nerfs auditifs) ${ }^{7}$.

Cette propriété du sémantème 'causer1' est prise en charge par son schéma actanciel. Ce schéma rend compte d'une caractéristique importante de la causation «physique ». En effet, une situation de causation d'un fait ' $\mathrm{Y}$ ' dans le monde réel repose sur un empilement de faits ' $\mathrm{X}_{i}$ ' qui sont tous des causes potentielles de ' $\mathrm{Y}$ '; leur nombre est potentiellement infini. Le locuteur est libre de choisir n'importe quel fait ' $\mathrm{X}_{k}$ ' comme la cause de ' $\mathrm{Y}$ ' et n'importe quel autre fait ' $\mathrm{X}_{i}$ ' assez contigu dans la suite de la pile comme une élaboration de la cause ' $\mathrm{X}_{k}$ '. Ces choix dépendent de la finesse avec laquelle le locuteur analyse la situation de causation dont il parle, c'est-à-dire de la profondeur de son analyse (cf. dans l'exemple (9), les voitures, le va-et-vient des voitures, le bruit de ce va-et-vient, l'impact de ce bruit sur les nerfs auditifs de Zoé, etc.). La structure tri-actancielle permet au sémantème 'causer1' de «se placer» à n'importe quel endroit de l'empilement de faits ' $\mathrm{X}_{i}$, en prenant comme cause n'importe quel ' $\mathrm{X}_{i}$ '.

En théorie, on peut continuer ce jeu de chaises musicales de façon illimitée. Ce procédé est aussi la source d'un type de paraphrase assez courant: 0a peut être paraphrasée par (9)b sans Élaboration, alors que (9)b avec Élaboration peut à son tour être paraphrasée par (9)c sans Élaboration, et ainsi de suite. Le fait d'avoir considéré

\footnotetext{
${ }^{7}$ La propriété d'admettre une telle "permutation» des arguments n'appartient pas exclusivement au sémantème 'causer1'. Ainsi, le sémantème 'changer' possède la même propriété : Jean a changé de visage $\sim$ Le visage de Jean a changé de forme $\sim$ La forme du visage de Jean a changé de rondeur $\sim$... Les signifiés des verbes de sentiment et d'attitude mentale possèdent une propriété semblable : Zoé admire Jean pour ses opinions profondes $\sim$ Zoé admire les opinions de Jean pour leur profondeur $\sim$ Zoé admire la profondeur des opinions de Jean $\sim \ldots$ (On observe néanmoins dans ce dernier cas un décalage sémantique : on peut admirer les opinions de Jean sans l'admirer lui-même.) Il existe encore d'autres verbes admettant à des degrés divers les mêmes "permutations » de ses arguments, comme, par exemple, Zoé a blessé Jean dans son orgueil Zoé a blessé l'orgueil de Jean, ... Les verbes (et les adjectifs) avec des sujets et des compléments « en cascade » ont attiré l'attention des linguistes à plusieurs reprises : cf. Apresjan 1974 : 259-260 et Guillet \& Leclère 1981 (surtout la page 120, où le terme «cascade» est introduit pour l'exemple suivant : Paris étonne Max par la diversité des attractions de ses cabarets $\sim$ Les cabarets de Paris étonnent Max par la diversité de leurs attractions $\sim$ Les attractions des cabarets de Paris étonnent Max par leur diversité).
} 
l'Élaboration de la Cause comme le troisième argument de 'causer1' permet de représenter la façon dont le locuteur «se déplace» dans l'empilement des causes potentielles et les paraphrases qui en découlent. Plus précisément, nous obtenons la règle d'équivalence sémantique suivante entre 'causer1' bi-actanciel et 'causer1' triactanciel (comme indiqué dans la note 3, le soulignement marque le nœud communicativement dominant du réseau en question $)^{8}$ :
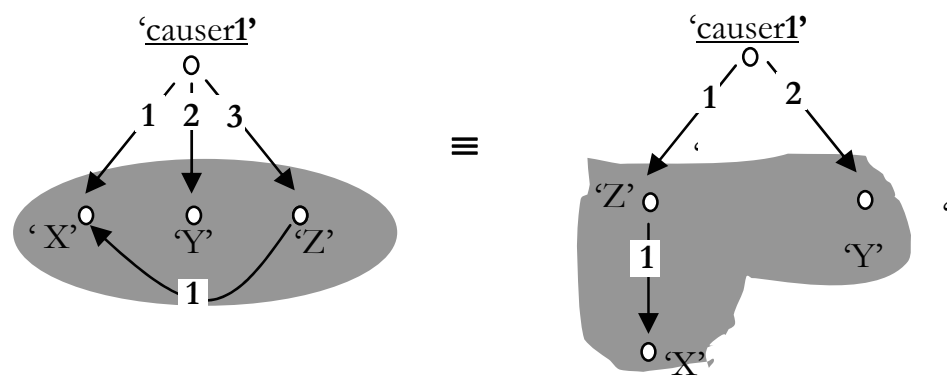

Figure 1 : Règle d'équivalence sémantique

pour les deux variantes « diathétiques » du sémantème 'causer1'

Dans une règle d'équivalence comme celle de la Fig. 1, les variables (ici, 'X', 'Y', ' $Z$ '), ainsi que toutes les dépendances qu'elles gouvernent (ici, la dépendance entre ' $Z$ ' et ' $\mathrm{X}$ ') appartiennent au contexte. Ce contexte, marqué par la zone ombrée, ne change pas avec l'application de la règle et est donc le même dans les configurations de gauche et de droite.

Quelques contraintes pèsent sur l'application de la règle de la Fig. 1. Par exemple, elle ne doit pas s'appliquer de droite à gauche à la structure sémantique [= SSém] de (10)a pour produire (10)b :

a. La mort [= 'Z'] de Jean a causé la tristesse [= 'Y'] de Zoé.

b. *Jean [= 'X'] a causé la tristesse [= 'Y'] de Zoé par sa mort [= 'Z'].

Dans le cas où $\mathrm{Z}$ réfère à une action involontaire de $\mathrm{X}$, la transformation, qui est formellement possible, produit un résultat sémantiquement mauvais, car la phrase obtenue $[=(10) \mathrm{b}]$ doit, mais ne peut pas, être interprétée comme comprenant 'causer2', comme le montre l'exemple (11):

$$
\begin{aligned}
& \text { a. Les caresses [= 'Z'] de Jean [= 'X'] ont causé l'irritation }[=' \mathrm{Y} \text { '] de Zoé. } \\
& \text { b. Jean }[=' \mathrm{X} \text { '] a causé l'irritation }[=' \mathrm{Y} \text { '] de Zoé par ses caresses [= 'Z']. }
\end{aligned}
$$

Les phrases (11)a et (11)b ne sont pas synonymes: (11)b, mais pas (11)a, dit que la causation est le résultat d'une action volontaire de Jean sur Zoé ou sur un tiers c'est-à-dire que c'est une causation2, alors que dans (11)a, on a une causation1.

\footnotetext{
${ }^{8}$ Une ébauche de cette règle est présentée dans Milićević $2003: 135$.
} 
L'impossibilité absolue de (10)b et l'impossibilité de (11)b avec le sens 'causer1' viennent de multiples contraintes sémantiques attachées à 'causer1'. Ces contraintes, qui interdisent l'emploi de 'causer1' dans certaines situations, ont été illustrées par la série d'exemples (2) et elles apparaissent dans la Fig. 2 plus loin. Pour les décrire formellement, il nous faut d'abord présenter la décomposition sémantique du sens 'causer1', car ces contraintes résultent en partie des éléments qui interviennent dans sa décomposition.

Comme nous l'avons déjà dit, nous pensons que le sémantème 'causer1' n'est pas une primitive sémantique, mais peut être décomposé en fonction de deux sémantèmes plus simples, 'agirI.1' et 'entraîner' (un sens particulier de ENTRAÎNER, voir p. 258).

\section{Le sémantème 'agirI.1'}

Le sémantème 'agirI.1' est un prédicat binaire: 'X agitI.1 sur Y'; nous le considérons comme une primitive sémantique. ' $\mathrm{X}$ ' n'est pas taxinomiquement contraint, alors que 'Y' est plutôt une entité physique (objet, individu, substance, ...), mais tout en pouvant être un fait (Les prix du pétrole agissent sur le taux de chômage). Le sémantème 'agirI.1' s'exprime en français surtout par le nom ACTIONI.1, comme dans les phrases suivantes :

(12) a. L'action prolongée des vagues sur la jetée n'a pas en d'effet.

b. La gravité exerce une action permanente sur tous les objets.

Le verbe AGIRI.1 correspondant est lexicalement plus contraint; ainsi, la phrase $0 \mathrm{a}$ est inacceptable, même si la phrase synonyme 0 a est normale :

(13) a. ?? Les vagues ont agi de façon prolongée sur la jetée (sans avoir d'effet).

b. La gravité agit de façon permanente sur tous les objets.

Le sémantème 'agirI.1' est essentiellement un construit théorique destiné à décrire 'causer1', car même dans des exemples comme $0 \mathrm{~b}$, il est difficile de ne pas considérer les effets permanents de la gravitation (le fait que les objets restent au sol et que les pommes pendent aux branches) et donc de rejeter l'expression d'une causation sous-jacente. Nous cherchons, avec notre sémantème 'agirI.1', à isoler et à nommer l'ensemble des conditions préalables à une causation1. Nous allons néanmoins tenter de justifier plus avant l'existence du sémantème 'agirI.1'.

Notre sémantème 'agirI.1' peut être contrasté avec l'acception usuelle la plus proche du vocable AGIR, que nous notons AGIRI.2 et qui, contrairement à 'agirI.1', décrit une action avec effet :

(14) a. L'acide a agi sur le métal.

b. Ce médicament n'agit pas sur ce malade.

c. Ses convictions ont agi sur son destin.

C'est justement l'observation du sémantème 'agirI.2' qui nous permet de justifier l'introduction du sémantème 'agirI.1'. En effet, la phrase Le médicament agit et 
sa négation, Le médicament n'agit pas, ou encore l'interrogation Le médicament agit-il ?, présupposent toutes que le médicament a été administré. C'est ce présupposé extrait de la définition de 'agirI.2' qui constitue le sémantème 'agirI.1'. Autrement dit, que $\mathrm{X}$ agisseI.2 sur $\mathrm{Y}$ ou que $\mathrm{X}$ n'agisseI.2 pas sur $\mathrm{Y}$, dans les deux cas, $\mathrm{X}$ agitI.1 sur $\mathrm{Y}$, c'està-dire qu'il y a eu une sorte de "contact» entre $\mathrm{X}$ et $\mathrm{Y}$ avec un transfert de "forces» de $\mathrm{X}$ vers $\mathrm{Y}$, et cela présente les conditions d'une causation 1 potentielle : 'X agitI.2 sur $\mathrm{Y}^{\prime} \equiv$ 'X agissantI.1 sur $\mathrm{Y}$, cette actionI.1 de X sur Y entraine un changement de l'état de $Y$ '.

Il est important de souligner que même si 'agirl.1' n'est pas à proprement parler le signifié d'une lexie du français, il s'agit bel et bien d'un sens du français et donc d'un objet linguistique à part entière. Cet objet n'est pas aussi facilement observable que peuvent l'être les sémantèmes ordinaires (les signifiés de lexies), mais il peut être mis en évidence, comme nous venons de le faire, par la manipulation d'autres sémantèmes. Apresjan (1995 : 481-482), qui est probablement le premier à avoir exhibé de tels sens, propose de les appeler des quarks sémantiques; nous les avons déjà mentionnés plus haut, dans la note 4.

Notons encore que notre numérotation des sémantèmes 'agirl.1' et 'agirI.2' n'est pas entièrement satisfaisante, car même si 'agirI.1' est plus " simple » que 'agirI.2' (puisque 'agirl.1' est inclus dans la décomposition de 'agirI.2'), c'est quand même AGIRI.2 qui est l'élément de base du vocable AGIR et c'est à partir de lui, par une construction théorique, que nous dégageons 'agirl.1'. Dans la suite, nous nous permettrons d'utiliser le sémantème 'agirl.1' dans nos décompositions sémantiques sans égard pour les contraintes lexicales du verbe AGIRI.1. (Cf. plus bas les remarques concernant la distinction entre les contraintes purement sémantiques sur les sémantèmes et les contraintes sémantico-lexicales sur les lexies correspondantes.)

Il existe par ailleurs une contrainte reliant les arguments ' $\mathrm{X}$ ' et ' $\mathrm{Y}$ ' de 'agirI.1' : 'X' n'est pas une actionI.1 sur 'Y' (pour 'actionII.1' - une action volontaire -, voir la section 4). Cette contrainte bloque les structures sémantiques des phrases telles que * La destruction de la ville a agi sur la ville, ${ }^{*}$ L'exécution du prisonnier a agi sur le prisonnier, etc. Une telle configuration de sémantèmes est redondante, puisque, comme nous le verrons plus loin en étudiant 'causer1', les noms EXÉCUTION ou DESTRUCTION (qui sont des déverbatifs de verbes causatifs) expriment déjà une actionI.1. Qui plus est, admettre ce type de configuration sémantique ouvrirait la porte à une récursion infinie : $\mathrm{X}$ agitI.1 sur $\mathrm{Y}$, l'actionI.1 de $\mathrm{X}$ sur $\mathrm{Y}$ agitI.1 sur $\mathrm{Y}$, l'actionI.1 sur $\mathrm{Y}$ de l'actionI.1 de X sur Y agitI.1 sur Y, etc. En conséquence, une SSém comprenant une sous-structure de la forme *'agirI.1 sur $\alpha$ agitI.1 sur $\alpha$ ' est interdite.

Par contre, nous autorisons ' $\mathrm{X}$ ' à être une actionI.1 (sur qqch), mais seulement quand ' $\mathrm{X}$ ' n'est pas une actionI.1 sur ' $\mathrm{Y}$ '. Plus précisément, si ' $\mathrm{X}$ ' est une actionI.1 sur autre chose que ' $\mathrm{Y}$ ', alors on peut mettre dans la SSém la configuration ' $\mathrm{X}$ agitI.1 sur Y' dans les deux cas suivants, où ' $\mathrm{Y}$ ' est un être animé :

\footnotetext{
${ }^{9}$ Une situation similaire existe en russe, où le sémantème 'agirI.1' correspond plutôt au signifié du verbe dérivé 'voz+dejstvovat", tandis que 'agirI.2' correspond au signifié du verbe simple 'dejstvovat". Comme le montre l'usage du préfixe (improductif) VOZ-, le russe a créé une désignation pour le sémantème problématique 'agirI.1/voz+dejstvovat" à partir de la désignation pour le sémantème 'agirI.2/ dejstvovat".
} 
- Soit 'Y' n'est pas le premier argument de 'X' et 'Y' perçoit 'X'. Par exemple, dans une situation décrite par la phrase La gifle donnée par Zoé à Félix a réveillé/étonné/fâché Jean, on mettra dans la SSém décomposée la configuration 'la gifle a agiI.1 sur Jean' (pour exprimer que la gifle a été perçue par Jean et que cette perception a agiI.1 sur lui). Par contre, si la gifle a été donnée à Jean lui-même, une telle configuration n'est pas permise.

- Soit 'Y' est le premier argument de 'X'. Par exemple, dans une situation décrite par la phrase Zoé a nettoyé la maison, ce qui l'a fatiguée on mettra dans la SSém décomposée 'le nettoyage de la maison par Zoé a agiI.1 sur Zoé'. (Mais, bien entendu, la configuration *`le nettoyage de la maison par Zoé a agiI.1 sur la maison’ est interdite.)

On comprendra mieux le sémantème 'agirI.1' en analysant son rôle dans la décomposition de 'causer1' proposée ci-dessous. Notons tout de suite que 'agirI.1' reflète des actions physiques, chimiques, psychologiques, etc. - directes ou indirectes. Il couvre également :

- des cas qui relèvent de la perception, comme dans la phrase La pâleur extrême de son visage cause l'inquiétude de ses parents, où la pâleur, perçue visuellement, agitI.1 ( $\equiv$ exerce une actionI.1) sur les parents ;

- des actionsI. 1 très indirectes, comme dans La guerre a provoqué la dégringolade $d u$ dollar, où la guerre agitI.1 sur la valeur du dollar à travers une chaîne assez longue d'actions $\mathbf{I} \mathbf{1}$ successives.

Concernant les actionsI.1 indirectes, soulignons le fait suivant :

Le sémantème 'agirI.1', propre au français, peut s'utiliser pour une actionI.1 indirecte (= une chaîne d'actionsI.1) seulement si le dernier « chaînon » de cette chaîne ne peut pas être mis en évidence pour des raisons linguistiques ou pragmatiques.

Ainsi, on ne peut pas dire que la carabine qui envoie une balle sur la cible agitI.1 sur la cible puisqu'on connaît le dernier chaînon, c'est-à-dire le premier argument de l'actionI.1 directe sur la cible : c'est la balle (cela découle des connaissances au sujet des carabines et des balles). Lorsqu'un avion s'écrase avec ses passagers à bord, le crash n'agitI.1 pas sur les passagers, bien que ce soit lui qui entraîne l'actionI.1 des forces meurtrières : par conséquent, on ne dira pas en français * Le crash de l'avion a tué tous les passagers. (Par contre, en anglais on dit sans problème The plane crash killed all the passengers, cf. (4). Donc, le sémantème anglais correspondant - 'actI.1' - n'a pas cette contrainte sémantique.)

\section{Le sémantème 'entraîner'}

Le sémantème 'entraîner' est un prédicat binaire - 'X entraîne $\mathrm{Y}$ ' - qui se décompose en une implication logique et un changement d'état dont ' $\mathrm{Y}$ ' est l'état final. 'X' et 'Y' sont nécessairement des faits; nous avons appelé les rôles sémantiques correspondants Déclencheur et Résultat. La description de 'entraîner' se présente donc ainsi : 
'X entraîne $\mathrm{Y}(\alpha)$ ' E 'si X, alors $\alpha$ change d'état et $\mathrm{Y}(\alpha)$ est l'état final de ce changement'.

Comme 'entraîner' dans le sens considéré ici est une composante de 'causer1', l'utilisation de 'causer1' implique l'utilisation possible de 'entraîner', mais pas l'inverse :

a. Le mariage de Zoé entraine qu'elle est majeure.

b. *Le mariage de Zoé la rend majeure (= *'le mariage de Zoé cause1 qu'elle est majeure')

Néanmoins, 'entrainner' n'est pas toujours substituable à 'causer1', car 'entraîner', mais pas 'causer1', exige que son premier argument soit un fait:

(16) a. L'acide a causé la mort de la grenouille.

b. *'L'acide a entrainé la mort de la grenouille.

Le sémantème 'entrainer' possède trois propriétés sémantiques importantes.

- Premièrement, le sémantème 'entraîner' est complétif, c'est-à-dire que 'entraîner' veut en fait toujours dire 'avoir entraîné' et l'expression *être en train d'entrainer est impossible ; l'emploi du verbe ENTRAÎNER au présent exprime toujours une affirmation générique et ne peut pas être interprété comme un vrai présent actuel.

- Deuxièmement, 'entraîner' est ponctuel, c'est-à-dire qu'il ne possède pas de durée et une expression du type *entrainer en $\mathrm{x}$ temps est impossible.

- Troisièmement, le sémantème 'entraîner' est implicatif, c'est-à-dire que si 'X a entrâné Y', alors 'Y a lieu'10.

Ces trois propriétés sont héritées par 'causer1' et, par ricochet, par 'causer2' : les deux sémantèmes sont aussi complétifs, ponctuels et implicatifs. (L'héritage des propriétés de complétivité, de ponctualité et d'implicativité par les verbes français ayant les sémantèmes 'causer1' et 'causer2' comme composante dominante n'est pourtant pas direct : il est assez complexe, en raison du caractère aspectuel des autres composantes, qui semblent interférer.)

On peut maintenant donner la décomposition, ou plutôt les décompositions, du sémantème 'causer1'. Ce sont en fait des règles d'équivalence, qui décrivent toutes les substitutions possibles pour 'causer1'.

Mais avant, une remarque s'impose au sujet du terme décomposition (sémantique). En général, la décomposition d'un sémantème ' $s$ ' se fait par une configuration de plusieurs sémantèmes (plus simples que 's'). Pour 'causer1', ce principe est également respecté : les décompositions que nous proposons comprennent toujours les sémantèmes 'agirI.1' et 'entraîner'. Néanmoins, le sémantème 'agirI.1' n'apparait pas forcément de façon explicite dans toutes les décompositions du sémantème 'causer1', car ce sémantème peut être "caché » à l'intérieur d'un des arguments de 'causer1'. C'est le cas lorsque ' $\mathrm{X}$ ' ou 'Z' est une actionI.1, c'est-à-dire que c'est un sémantème ayant 'agirI.1' comme nœud dominant de sa décomposition (cf. règles 2 et 4).

\footnotetext{
10 On appelle implicatif un verbe transitif qui, utilisé au passé composé, implique que l'état visé par l'action sur Y décrite par le verbe a été atteint. Par exemple, TUER est un verbe implicatif, car l'expression $X$ a tué $Y$ permet de conclure que $Y$ est mort ; par contre, TIRER [sur qqn] n'est pas implicatif, car l'expression $X$ a tiré sur $Y$ ne permet pas de conclure que $Y$ a été touché.
} 
Les règles qu'on voit dans la Fig. 2 permettent deux choses : soit, si on les applique de gauche à droite, elles développent le réseau sémantique qui contient le sémantème 'causer1'; soit, si on les applique de droite à gauche, elles permettent de réduire un réseau sémantique qui contient une décomposition du sémantème 'causer1'.

Rappelons que nous considérons deux variantes du sémantème 'causer1' - biactancielle et tri-actancielle (liées par la règle d'équivalence de la Fig. 1). Les quatre décompositions présentées ci-dessous sont donc équivalentes les unes aux autres et épuisent tous les cas de figure possibles. Insistons sur le fait qu'il s'agit bel et bien d'un seul sémantème 'causer1', qui apparaît sous deux formes diathétiques et admet quatre décompositions.

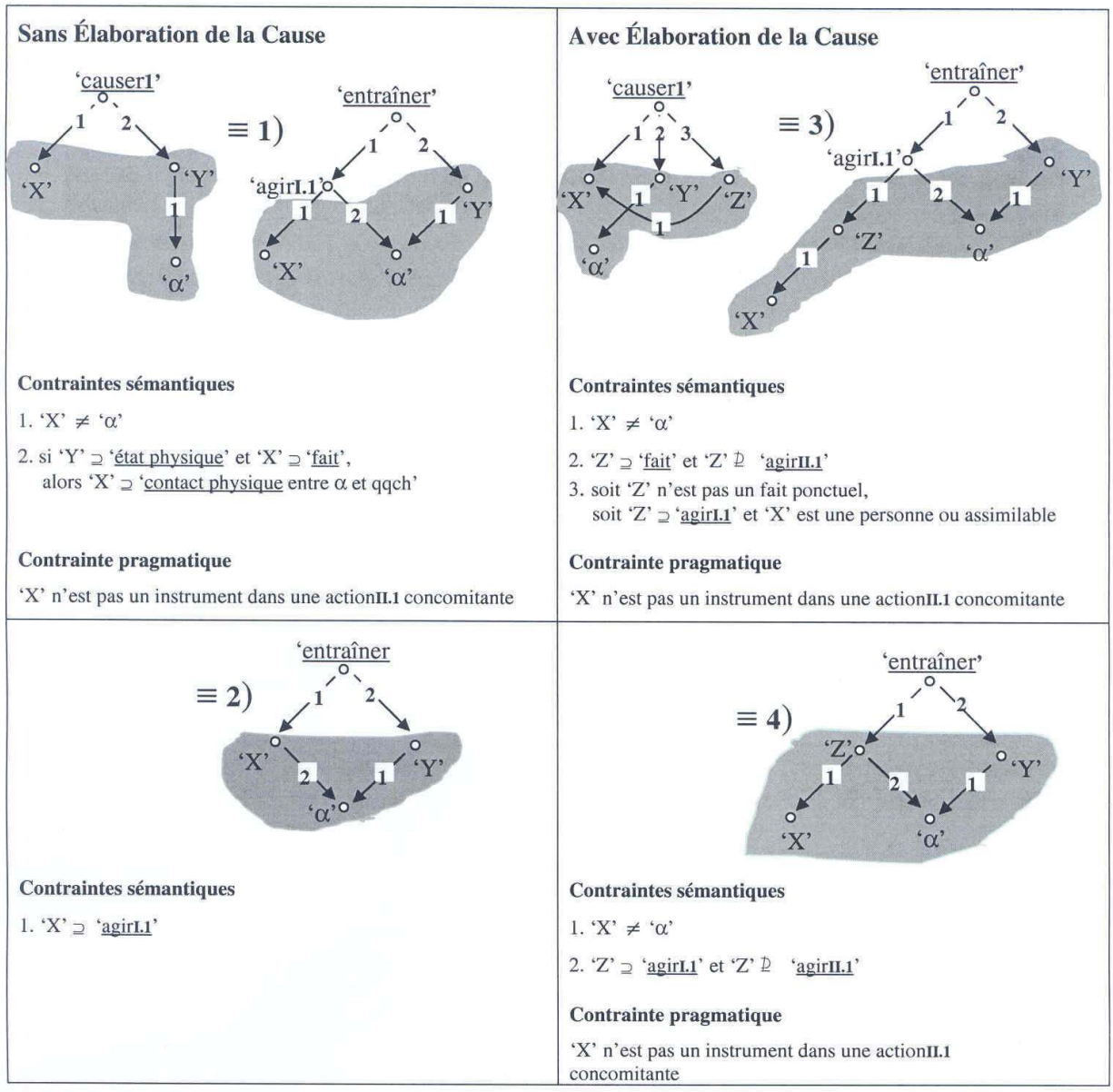

Figure 2 : Les quatre décompositions possibles du sémantème 'causer1'

Nous allons maintenant illustrer et commenter les quatre cas de la Fig. 2. Mais avant, précisons la distinction entre Cause et Déclencheur (voir Section 1) : la Cause est le premier argument de 'causer1', tandis que le Déclencheur est le premier argument de 'entraîner' dans la décomposition de 'causer1'. 


\section{(17) Règle 1}

a. La course a causé la fatigue de Zoé.

(Cause : Zoé a couru ; Déclencheur : la course a agiI.1 sur Zoé.)

b. Sa grande taille/Le fait d'être mère cause le bonheur de Zoé.

(Cause : Zoé est grande/Zoé est mère ; Déclencheur : sa grande taille/le fait d'être mère agitI.1 sur Zoé, car elle en est consciente.)

c. Fumer tue/cause la mort.

(Cause : des personnes fument; Déclencheur : fumer agitI.1 sur la personne qui fume.)

d. L'acide a causé la dissolution du tissu.

(Cause : l'acide ; Déclencheur : l'acide a agiI.1 sur le tissu.)

e. Les transparents causent des dégâts à (= abiment) la photocopieuse.

(Cause: les transparents; Déclencheur: les transparents agissentI.1 sur la photocopieuse qui les copie.)

f. La balle a causé la mort de la grenouille.

(Cause : la balle ; Déclencheur : la balle a agil.1 sur la grenouille.)

g. La poutre a causé la mort de la grenouille (en lui tombant dessus/*par sa cbute). (Cause: la poutre; Déclencheur: la poutre a agiI.1 sur la grenouille. Attention, la chute de la poutre n'est pas l'Élaboration de la Cause, mais un modificateur circonstanciel: la chute comme telle n'a pas agit.1 sur la grenouille.)

h. L'explosion de la bombe a causé la destruction du (= a détruit le) pont.

(Cause : la bombe a explosé ; Déclencheur : l'explosion a agil.1 sur le pont.)

i. La bombe a causé la destruction du (= a détruit le) pont.

(Cause : la bombe ; Déclencheur : la bombe a agit.1 sur le pont.) ${ }^{11}$

11 On comparera les deux exemples précédents avec L'explosion du dépôt de munitions a détruit le pont vs * Le dépôt de munitions a détruit le pont. Dans une situation où un dépôt de munitions a explosé et cette explosion a détruit le pont, on ne peut pas dire que le dépôt a agii.1 sur le pont. Par contre, on dit sans problème qu'une bombe, en explosant, a agi.1.1 sur le pont. Cette propriété du lexème BOMBE est prise en charge par la composante sémantique (dans sa définition) 'destinée à exploser pour agirl.1 sur Y et détruire Y'. DÉPÔT DE MUNITIONS ne contient rien de semblable dans sa définition. En même temps si un avion tombe sur un pont, on pourra dire L'avion a détruit le pont, même si la définition de AVION, tout à fait comme celle de DÉPÔT DE MUNITIONS, ne contient pas la composante 'destiné à exploser ...' La différence pertinente est que l'avion en s'écrasant sur le pont entre en contact avec le pont, tandis que le dépôt de munitions en explosant n'entre pas en contact avec le pont. Pour montrer l'importance de la composante 'destiné à exploser ...', on peut même citer la paire minimale * La voiture a détruit le pont en explosant vs La voiture piégée garée depuis hier a détruit le pont. Plus généralement, la configuration sémantique 'objet X cause1 Y' est permise si nos connaissances 
Sylvain Kahane \& Igor Mel’čuk

j. La cigarette cause la mort/ tue.

(Cause : la cigarette; Déclencheur : la cigarette agitI.1 sur les personnes qui la fument.) $)^{12}$

k. La mort du tyran a causé une joie immense à la population.

(Cause : le tyran est mort; Déclencheur: la mort du tyran a agiI.1 sur la population - la population a appris que le tyran est mort.)

1. La gifle de Zoé à Jean a causé l'irritation de Max.

(Cause : Zoé a giflé Jean ; Déclencheur : la gifle de Zoé à Jean a agiI.1 sur Max - par exemple, Max a vu que Zoé a giflé Jean.)

m. * Le tuyau a causé sa (propre) rupture.

(Cause : le tuyau; Déclencheur : le tuyau a agiI.1 sur lui-même, par exemple en frottant contre la cloison. La phrase est bloquée par la contrainte sémantique 1 ; on peut contraster cet exemple inacceptable avec Le tuyau a causé la rupture de la cloison et Le frottement du tuyau contre la cloison a causé sa rupture [la rupture du tuyau ou de la cloison].)

n. *La chute/*La course a causé à Zoé une blessure à la jambe.

(Cause : Zoé est tombée/Zoé a couru; Déclencheur: la chute/la course a agiI.1 sur Zoé. Ces phrases sont bloquées par la contrainte sémantique 2 stipulant que si ' $\mathrm{Y}$ ' est un état physique, alors ' $\mathrm{X}$ ' doit inclure la composante 'contact physique entre $\alpha$ et qqch.', cf., d'une part, Ce choc brutal lui a causé une blessure à la jambe et, d'autre part, La chute/La course lui a causé une peur bleue.)

o. Le passage du camion a causé la rupture de la passerelle/? des fissures dans la passerelle. (Cause : le camion est passé sur la passerelle; Déclencheur: le passage du camion a agiI.1 sur la passerelle. Le sémantème 'rupture' ne réfère pas à un état physique, mais à un événement, et la phrase est permise ; par contre, les

linguistiques et encyclopédiques sur $\mathrm{X}$ sont telles qu'on puisse inférer que le fonctionnement de $\mathrm{X}$ peut produire un phénomène $\mathrm{X}^{\prime}$ qui cause $\mathbf{Y} \mathrm{Y}$. Il s'agit d'une métonymie sémantique :

(i) Le ventilateur a causé du désordre sur mon bureau.

(Déclencheur : le souffle du ventilateur a agiI.1 sur les papiers posés sur mon bureau.)

(ii) L’hélicoptère a détruit les fleurs en décollant. (Déclencheur : le souffle de l'hélicoptère a agiI.1 sur les fleurs.)

(iii) L'usine de retraitement chimique a tué tous les poissons du lac.

(Déclencheur : les eaux usées de l'usine ont agiI.1 sur les poissons.)

12 La représentation sémantique de la phrase La cigarette tue est quasi-équivalente à celle de la phrase Fumer (des cigarettes) tue. Le sémantème 'cigarette (de X)' contient dans sa décomposition 'destiné à être fumé par X pour agirI.1 sur X en procurant du plaisir à X'. Par conséquent, 'X fume des cigarettes' $\Rightarrow$ 'les cigarettes agissentI.1 sur X'. Inversement, par défaut, on peut conclure que 'les cigarettes agissentI.1 sur $\mathrm{X}$ ' $\Rightarrow$ 'X fume des cigarettes' ; mais cette dernière implication peut être bloquée par la spécification d'une situation qui la contredit (par exemple, on pourra dire La cigarette tue pour évoquer la consommation passive du tabac par des enfants de fumeurs ou les règlements de comptes entre trafiquants de cigarettes). 
'fissures' représentent un état physique, alors que l'actionI.1 du passage, bien que présupposant le contact physique entre le camion et la passerelle, n'a pas la composante 'contact' comme composante centrale, et en vertu de la contrainte sémantique 2 la phrase résultante n'est pas parfaite; par contre, une phrase telle que Le choc du camion contre la passerelle a causé des fissures dans celle-ci ne pose pas de problèmes.)

\section{p. Le marteau a causé de grands dégâts à la véranda.}

(Cause: le marteau; Déclencheur: le marteau a heurté la véranda. Cette phrase est correcte s'il s'agit d'un marteau tombé sur la véranda par inadvertance, mais elle est bloquée - par la contrainte pragmatique - si le marteau est un instrument de quelqu'un dans une actionII.1 concomitante, par exemple, si Zoé frappe la véranda avec le marteau.)

\section{(18) Règle 2}

a. Les caresses de Zoé ont causé l'irritation de Jean.

$\left(\right.$ Cause $=$ Déclencheur : Zoé a caressé Jean ${ }^{13}$.)

b. Le coup de Zoé lui a causé une blessure à l'épaule.

(Cause $=$ Déclencheur : Zoé l'a frappé.)

c. La pression de la poutre a causé la mort de la grenouille.

(Cause $=$ Déclencheur $:$ la poutre appuie sur la grenouille. $)$

d. *Le fait que la maison soit proche du métro cause son prix élevé.

(Cause $=$ Déclencheur $:$ la maison est proche du métro; le fait d'être proche du métro n'est pas une actionI.1, ce qui est exigé par la contrainte sémantique 1. Par contre, avec cette configuration sémantique, on peut produire une phrase comme Le fait que la maison soit proche du métro implique son prix élevé. C'est donc bien l'absence du sémantème 'agirI.1' qui bloque l'introduction du sémantème 'causer1'. On ne peut même pas produire une configuration où la sous-règle 1 s'appliquerait parce que le fait que la maison soit proche du métro ne peut pas agirI.1 sur la maison ; ceci est interdit par les contraintes sémantiques sur le sémantème 'agirI.1' : une propriété d'une chose - mais pas d'un être animé - ne peut pas agirI.1 sur cette chose.)

Les règles 3 et 4 sont parallèles aux règles 1 et 2 , à l'expression de l'Élaboration de la Cause près.

\footnotetext{
13 Si Zoé a caressé quelqu'un d'autre que Jean, nous ne sommes pas dans ce cas : l'irritation n'est pas entraînée par les caresses elles-mêmes, mais par la perception par Jean de ces caresses et cette situation est couverte par la règle 1 . Si Zoé a caressé Jean, nous avons 'caresser' qui comprend 'action sur Jean', et dans la structure sémantique, ' $\mathrm{X}$ ' ne peut pas être le premier argument de 'agirI.1'; comme résultat, la règle 1 devient inapplicable.
} 


\section{(19) Règle 3}

a. Les voitures causent l'irritation de (= irritent) Zoé par leur va-et-vient.

(Cause: les voitures; Déclencheur: Zoé perçoit que les voitures vont et viennent, et cela agitI.1 sur elle.)

b. La bombe a causé la destruction du (= a détruit le) pont en explosant.

(Cause : la bombe ; Déclencheur : l'explosion de la bombe a agiI.1 sur le pont.)

c. ${ }^{*}$ La voiture a causé une bosse en heurtant le mur.

(Cause : la voiture ; Déclencheur : la voiture a heurté le mur, ce qui a agiI.1 sur elle ; cf. Le choc a causé une bosse à la voiture. La phrase Oc est bloquée par la contrainte sémantique 1 - il s'agit d'une bosse à la voiture elle-même, alors qu'il est possible de dire La voiture a causé une bosse au bus en le heurtant de plein fouet. Rappelons que non seulement ' $\mathrm{X}$ ' doit être différent de ' $\alpha$ ', mais aussi du premier argument de ' $\alpha$ ', ou même du premier argument de celui-ci, etc. : *La voiture a causé une fuite au carburateur en beurtant le mur, quand il s'agit du carburateur de la voiture elle-même ; ceci est la conséquence de la métonymie de possession, dont on a déjà parlé.)

d. Le marteau a causé l'effroi de (= a effrayé) Jean en heurtant la véranda.

(Cause : le marteau; Déclencheur: Jean a vu/entendu le marteau heurter la véranda. Cette phrase est bonne, si le marteau est tombé sur la véranda par hasard, mais elle est bloquée par la contrainte pragmatique si le marteau était un instrument de quelqu'un dans une actionII.1 concomitante - par exemple, si Zoé a frappé la véranda avec le marteau.)

e. "Zoé a causé l'effroi de (= a effrayé) Jean en frappant la véranda avec un marteau.

(Cause : Zoé ; Déclencheur : Jean a vu/entendu Zoé frapper la véranda avec un marteau, et cela a agiI.1 sur lui ; ' $Z$ ' = 'frapper' contient 'agirII.1', ce qui est interdit par la contrainte sémantique 2 , et donc cette phrase ne sera pas produite par la règle 3 ; elle le sera par la règle qui introduit 'causer2', voir plus loin, la section 3, Fig. 7.)

f. Cette voiture cause des soucis à Zoé par son manque de stabilité/*par sa dégradation trop rapide.

(Cause : cette voiture; Déclencheur: Zoé perçoit que cette voiture manque de stabilité/se dégrade trop rapidement, et cela agiI.1 sur elle ; 'manquer de stabilité' n'est pas un événement, tandis que 'se dégrader' est un événement qui n'est pas une actionI.1 et donc la phrase est bloquée par la contrainte sémantique 3 .)

g. La forêt cause l'inquiétude de (= inquiète) Zoé par son état catastrophique/*par son incendie.

(Cause : la forêt ; Déclencheur: Zoé perçoit que la forêt se trouve dans un état catastrophique/a brûlé, et cela agiI.1 sur elle; '(être dans) un état 
catastrophique' n'est pas un événement, tandis que 'brûler' est un événement qui n'est pas une actionI.1, et donc la phrase est bloquée par la contrainte sémantique 3.)

h. Zoé a causé l'inquiétude de ses parents en étant malade plusieurs semaines/*en tombant malade.

(Cause: Zoé; Déclencheur: les parents de Zoé ont appris qu'elle était malade/était tombée malade ; ' $Z$ ' = 'être malade' n'est pas un événement mais un état, tandis que ' $\mathrm{Z}$ ' = 'tomber malade' est un événement mais pas une actionI.1, ce qui est interdit par la contrainte sémantique 3.)

i. *L'acide a causé l'étonnement de Zoé par la dissolution du tissu.

(Cause : l'acide; Déclencheur: Zoé perçoit la dissolution du tissu, et cela agitI.1 sur elle. Mais l'acide n'est pas assimilable à une personne, et la contrainte sémantique 3 bloque la phrase.)

j. "Zoé s'est causé une blessure à la main en heurtant le mur.

(Cause : Zoé ; Déclencheur : Zoé a heurté le mur. Cette phrase est possible dans une situation où Zoé est responsable de cet événement - elle balaie, elle joue, etc., mais alors on a affaire à 'causer2' et cette configuration n'est pas traitée par la règle 3 - en raison de la contrainte sémantique 2, qui dit que 'Z' ne contient pas 'agirII.1'. Par contre, la phrase devient inappropriée si Zoé n'est pas responsable de sa blessure - par exemple, elle a été poussée contre le mur ou bien elle était inconsciente, et la phrase n'est pas non plus produite par la règle 3 en raison de la contrainte sémantique 3 , tout à fait comme dans l'exemple 0e.)

\section{(20) Règle 4}

a. La balle a causé la mort de la grenouille en lui éclatant la cervelle.

(Cause : la balle ; Déclencheur = Élaboration : la balle a éclaté la cervelle de la grenouille.)

b. ${ }^{\#}$ Zoé a cassé la véranda en la frappant avec un marteau.

(Cause : Zoé ; Déclencheur : Zoé a frappé la véranda avec un marteau; dans cette phrase 'casser' contient 'causer2' et la phrase est bloquée par la contrainte sémantique 1 , parce que ' $Z$ ' = 'frapper' contient 'agirII.1'.)

c. ${ }^{\#}$ Le marteau a cassé la véranda en la beurtant.

(Cause: le marteau; Déclencheur: le marteau a heurté la véranda ; cette phrase est bloquée - par la contrainte pragmatique - si le marteau est un instrument de quelqu'un dans une actionII.1 concomitante, par exemple, si Zoé frappe la véranda avec le marteau.)

Illustrons maintenant l'application des règles de décomposition de la Fig. 2, sur une structure sémantique [dorénavant SSém]. Considérons la SSém suivante sur laquelle on souhaite appliquer une règle de réduction : 


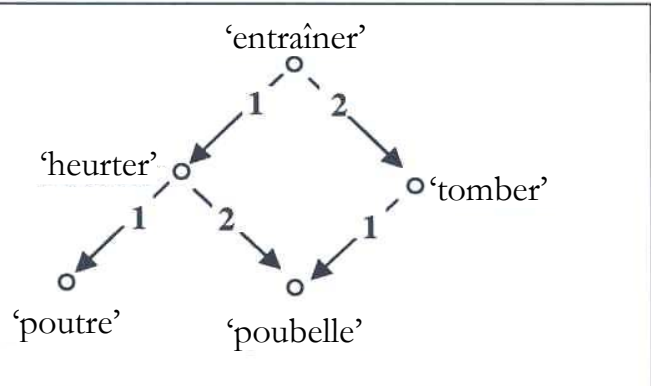

Figure 3 : Une SSém comprenant une décomposition de 'causer1'

Deux des règles de décomposition de 'causer1' - les règles 2 et 4 - peuvent s'appliquer sur cette SSém. En les appliquant de droite à gauche, on obtient deux SSém « réduites » correspondant aux phrases (21):

(21) a. Le choc de la poutre contre la poubelle a causé la chute de la poubelle.

b. La poutre a causé la chute de la poubelle en l'heurtant.

Pour appliquer la règle 2, 'heurter' est pris comme ' $\mathrm{X}$ ', tandis que l'application de la règle 4 nécessite de prendre 'poutre' comme ' $\mathrm{X}$ ' et 'heurter' comme ' $\mathrm{Z}$ '.

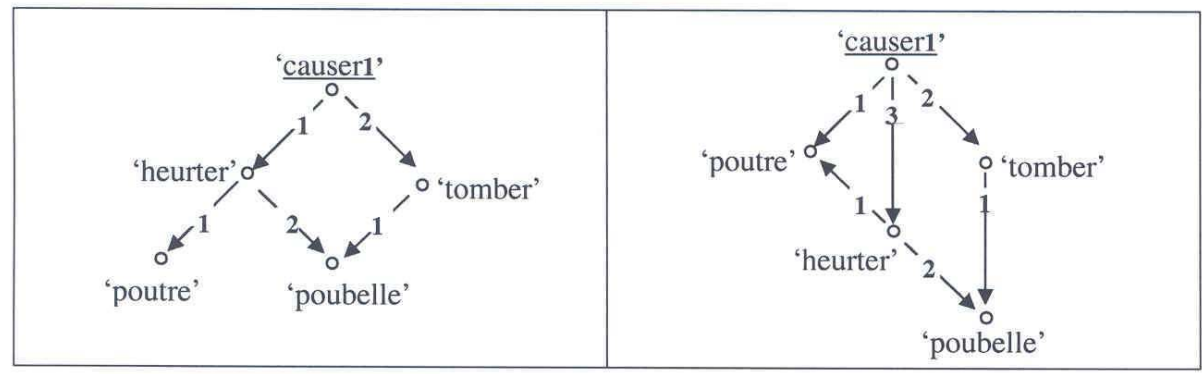

Figure 4 : Deux SSém équivalentes comprenant 'causer1'

La possibilité d'appliquer deux règles de décomposition différentes de 'causer1' à une même SSém tient au caractère escamotable du troisième argument 'Z' de 'causer1' : on peut, pour une même SSém de départ, considérer que la cause est ' $Z$ ' ou bien escamoter le troisième argument et retenir uniquement deux arguments pour 'causer1'.

Notons encore que la règle d'équivalence de la Fig. 1 est une conséquence des règles de décomposition de 'causer1' (Fig. 2) : l'équivalence des 'causer1' bi-actanciel et tri-actanciel s'obtient par la composition de deux règles de décomposition de 'causer1'. Par exemple, en décomposant 'causer1' tri-actanciel par la règle 3, puis en le recomposant par la règle 1 , on obtient 'causer1' bi-actanciel (au passage le premier argument de 'causer1' sera devenu ' $Z$ '). Il est normal que nous obtenions la règle d'équivalence de la Fig. 1, puisque nous avons élaboré nos règles de décomposition de 'causer1' avec l'idée que les 'causer1' bi-actanciel et tri-actanciel sont équivalents. 
Le fait que 'causer1' a quatre décompositions ne signifie cependant pas qu'il est «polysémique ». En fait, les quatre décompositions sont des variantes d'expression d'un même contenu sémantique :

$$
\text { 'une actionI.1 sur } \alpha \text { entraîne } Y \text { de } \alpha \text { '. }
$$

La première complication vient du fait que cette actionI.1 n'est pas toujours explicite : le premier argument du sémantème 'entrainer' n'est pas nécessairement le sémantème 'agirI.1' et peut être une actionI.1 plus spécifique ('pousser', 'heurter', 'renverser', etc.). La deuxième complication est due à la flexibilité extrême du sémantème 'causer1', qui :

1) est soit bi-actanciel, soit tri-actanciel,

2) prend comme premier argument soit l'actionI.1, soit l'agent de cette actionI.1, et

3) admet en tant qu'actionI.1 sur ' $\alpha$ ' soit une actionI.1 véritable, soit, comme nous l'avons déjà mentionné, la perception (par l'être animé ' $\alpha$ ') d'une actionI.1.

De façon plus formelle, les quatre décompositions de 'causer1' représentent les quatre solutions minimales d'un même ensemble de contraintes. Une configuration de sémantèmes est une solution d'un ensemble de contraintes si elle satisfait toutes les contraintes ; elle est minimale s'il n'existe pas de solution avec un plus petit nombre de sémantèmes. Dans le cas particulier de la décomposition de 'causer1', le caractère minimal d'une solution dépend des valeurs réelles des variables ' $\mathrm{X}$ ', 'Y' et ' $\mathrm{Z}$ ': par exemple, la solution 2 (= règle 2 de la Fig. 2) n'est possible que si ' $\mathrm{X}$ ' et ' $\mathrm{Y}$ ' ont le même premier argument.

Voici l'ensemble de contraintes décrivant les quatre décompositions de 'causer1'.

Une décomposition $D$ de 'causer1' doit posséder les quatre propriétés suivantes :

1. La composante communicativement dominante de $D$ est 'entraîner'; le deuxième argument de 'entraîner' est 'Y', qui a lui-même comme premier argument ' $\alpha$ '.

2. Le premier argument de 'entraîner', que nous notons ' $\beta$ ', est un sémantème qui contient 'agirI.1' comme nœud dominant ; si ' $\beta$ ' n'est pas spécifié par ailleurs, il sera donc égal à 'agirI.1'.

3. ' $\beta$ ' domine ' $\mathrm{X}$ ' par une chaîne de dépendances sémantiques d'étiquette 1 , qui représente la relation "prédicat - premier argument». (Cette chaîne est indiquée dans le schéma de la Fig. 5 par une flèche en pointillé ; elle peut être vide, auquel cas ' $\beta$ ' et ' $\mathrm{X}$ ' coïncident.)

4. Si 'causer1' est tri-actanciel, $D$ contient un sémantème ' $Z$ ', qui a ' $\mathrm{X}$ ' comme premier argument et qui est lui-même dominé par ' $\beta$ '.

Ces propriétés constituent les contraintes structurelles sur $D$. On peut représenter cet ensemble de contraintes par le schéma de la Fig. 5. (Les parenthèses autour d'un fragment de graphe indiquent son caractère optionnel.) 
Aux contraintes structurelles s'ajoutent cinq contraintes sémantiques que nous donnons dans la Fig. 5 et commenterons ci-après. Les contraintes structurelles caractérisent la nature même de la décomposition de 'causer1' (la «substance » de 'causer1'), alors que les contraintes sémantiques contrôlent l'applicabilité de cette décomposition à des structures sémantiques.

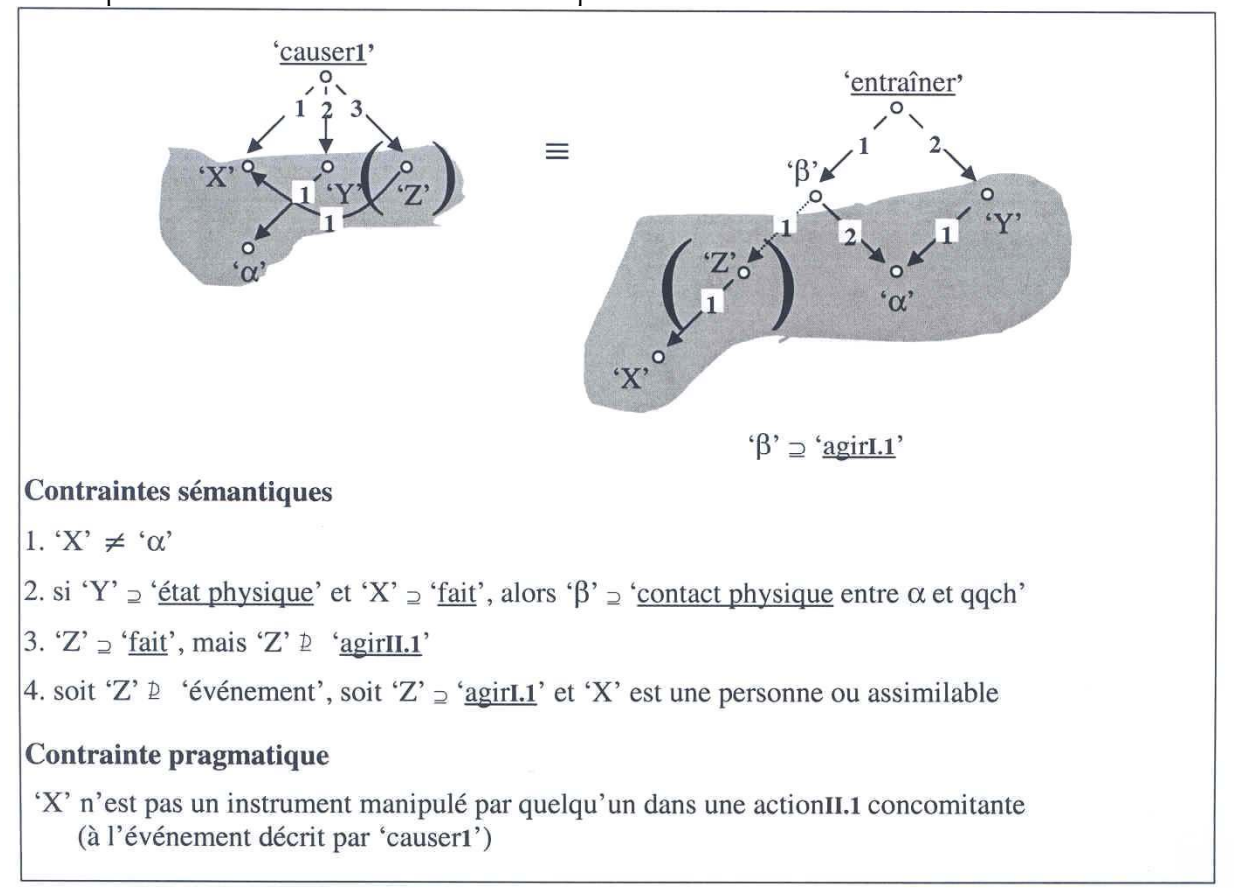

Figure 5 : Schéma généralisé de décomposition de 'causer1'

Le schéma de la Fig. 5 est en fait une autre façon de représenter les règles de décomposition de 'causer1' de la Fig. 2.

Dans une SSém donnée où les arguments de 'causer1' sont instanciés, une seule décomposition de 'causer1' est possible : il s'agit de la solution minimale de l'ensemble de contraintes présenté ci-dessus. Le résultat dépend donc de la possibilité pour ' $\beta$ ' de coïncider ou non avec 'X' ou 'Z'. Par exemple, dans le cas de 0a, 'Z' n'est pas spécifié et l'argument ' $\mathrm{X}$ ' (= 'les caresses de Zoé') est une actionI.1 sur le premier argument ' $\alpha$ ' (= 'Jean') de 'Y' (= 'irritation') ; 'X' et ' $\beta$ ' peuvent donc coïncider, et la minimalité de la solution les fera ainsi coïncider.

Commentons maintenant les quatre contraintes sémantiques et la contrainte pragmatique qui apparaissent dans (le schéma généralisé de) la décomposition de 'causer1' (Fig. 5). Les sémantiques assurent la bonne formation de la SSém, tandis que la contrainte pragmatique porte sur la correspondance entre la situation « réelle » de causation et la SSém.

La contrainte 1 décrit l'impossibilité pour ' $\mathrm{X}$ ', le premier actant de 'causer1', de causer1 un changement à soi-même. Cette contrainte est illustrée par les exemples (17)n et (19)c. 
La contrainte 2 stipule que si ' $\mathrm{Y}$ ' est un état physique et ' $\mathrm{X}$ ' est un fait, alors ' $\mathrm{X}$ ' présuppose un contact physique entre ' $\alpha$ ' et quelque chose; elle assure le bon traitement des phrases (19) f-k, ainsi que (22):

(22) a. La valise [= 'X'] a causé une blessure [= 'Y'] à Zoé en tombant [= 'Z'].

b. ${ }^{*}$ La chute $[=$ 'X'] de la valise a causé une blessure [= 'Y'] à Zoé.

c. Le frottement $[=$ 'X'] de la valise a causé une blessure $[=$ 'Y'] à Zoé.

La phrase (22)b - ou plus exactement la SSém correspondante - est bloquée par la contrainte 2 : ' $\mathrm{Y}$ ' = 'blessure' est un état physique et ' $\mathrm{X}$ ' = 'chute' = 'tomber' est un fait, mais ce sens n'inclut pas le sens de contact physique entre ' $\alpha$ ' = 'Zoé' et quelque chose ; il y a bien un contact entre Zoé et la valise qui lui tombe dessus (d'où l'acceptabilité de (22)a), mais ce contact est seulement une résultante de la chute. La phrase (22)c ne pose pas de problème, car ' $\mathrm{X}$ ' = 'frottement' inclut la composante 'contact physique'.

Cependant, la contrainte 2 est trop approximative. Premièrement, elle permet les deux phrases en (23) et ne rend pas compte du contraste observé :

a. L'explosion a causé la destruction du pont/de la moitié de la ville.

b. ' ${ }^{*}$ L'explosion a cassé toutes les vitres de l'hôpitall abîmé plusieurs arbres.

Pour autoriser (23)a, tout en continuant à bloquer $0 \mathrm{~b}$, il faudrait préciser les caractéristiques sémantiques du fait ' $\mathrm{X}$ ' et de l'état ' $\mathrm{Y}$ ', par exemple, en relâchant la contrainte pour des événements "actifs» comme une explosion, qui peuvent causer des états physiques "globaux » (détruire un pont, raser une ville, ravager une région, etc.), mais pas «locaux » (casser une vitre, abîmer un arbre, etc.). Les conditions sémantiques ne suffiront pas et il faudra s'appuyer sur des conditions pragmatiques.

Deuxièmement, la contrainte 2 ne couvre pas les conditions qui peuvent exister avec d'autres types de ' $\mathrm{Y}$ ', par exemple, un lien spécial entre ' $\mathrm{X}$ ' et ' $\mathrm{Z}$ ' lorsque ' $\mathrm{Y}$ ' est un état psychique :

a. * La valise a causé l'irritation de Zoé en tombant/par sa chute.

b. La chute de la valise a causé l'irritation de Zoé.

c. La valise cause livrritation de Zoé par son aspect moche/par sa présence intempestive.

La contrainte 2 permet les trois phrases. Il nous faudra donc une contrainte additionnelle qui pourrait être : si ' $\mathrm{Y}$ ' est un état psychique et ' $\mathrm{X}$ ' est une entité passive, alors 'Z' n'est pas un événement.

Troisièmement, il y a un problème qui peut être illustré par le contraste suivant :

a. ${ }^{*}$ La chute du pot de fleurs a causé la mort de la grenouille.

(Cause $=$ Déclencheur $:$ le pot de fleurs est tombé sur la grenouille.)

b. La chute de la coupole a causé la mort de plusieurs personnes.

(Cause $=$ Déclencheur $:$ la coupole est tombée sur ces personnes.) 
Strictement parlant, 'X tombe sur Y' n'inclut pas la composante ' $\mathrm{X}$ agitI.1 sur $\mathrm{Y}$ ' ('Y' n'est pas le Patient de 'tomber', mais le Point final du déplacement de 'X'). Les phrases (25) ne peuvent donc pas être produites par la sous-règle 2, qui exige que le Déclencheur soit une actionI.1, alors qu'ici, c'est un événement. La phrase (25)b sera produite par la règle 3 à partir d'une SSém où le Déclencheur est une actionI.1 de la chute de la coupole sur les personnes. L'inacceptabilité de la phrase (25)a induit que la SSém où la chute du pot de fleurs agitI.1 sur la grenouille n'est pas possible dans la situation où un pot de fleurs tombe sur la grenouille et entraine sa mort. Le contraste entre (25)a et (25)b relève donc à notre avis de la correspondance entre la représentation conceptuelle et la représentation sémantique et s'expliquerait par des considérations pragmatiques : la chute d'une coupole sur les personnes présentes dans le bâtiment implique fortement l'action I.1 de cette coupole sur eux, alors que la chute d'un objet isolé, ici un pot de fleurs, n'agitI.1 pas en tant que tel sur la «destination» de cette chute.

Les contraintes 3 et $\mathbf{4}$ stipulent que 'Z' est un fait, mais n'est ni une actionII.1, ni un événement autre qu'une actionI.1. Lorsque ' $Z$ ' est une actionII.1 (une action volontaire d'un animé ' $\mathrm{X}$ ' sur ' $\mathrm{Y}$ '), il s'agit d'un autre type de causation, exprimé par le sémantème 'causer2'; nous développerons ce point dans la section suivante. Disons ici seulement que cette sous-condition sur le type d'action effectuée par ' $\mathrm{X}$ ' nous semble représenter la frontière entre deux types de causation: 'causer1' vs 'causer2'. Par exemple, si Pierre a renversé le vase en balayant la pièce, bien que la causation du renversement puisse être tout à fait involontaire, le fait que l'effet - la chute du vase résulte d'une action volontaire de Pierre suffit pour qu'il soit tenu pour responsable ; il est le Causateur et pas la Cause de la chute du vase. Par contre, si Pierre a été poussé par Jean et que sa chute a entraîné celle du vase, on ne pourra le tenir pour responsable et on ne dira pas ${ }^{\#}$ Pierre a renversé le vase en tombant, mais plutôt quelque chose comme Le vase a été renversé quand Pierre est tombé.

La deuxième partie de la contrainte 4 ('Z' n'est pas un événement autre qu'une actionI.1) est justifiée par les phrases (26):

a. *Jean a causé mon désespoir en mourant/en tombant amoureux de cette fille. vs

La mort de Jean/Le fait que Jean soit tombé amoureux de cette fille a causé mon désespoir. ${ }^{14}$

b. * La forêt a causé mon désespoir en brûlant.

vs

L'incendie de la forêt a causé mon désespoir.

Mourir, tomber amoureux ou brûler sont des événements qui ne sont pas des actionsI.1. Par contre, pour un événement qui est une actionI.1, comme quitter

14 En russe, les phrases signifiant 'En tombant amoureux de cette fille, Jean a causé mon désespoir' ou 'Par sa naissance, cet enfant m'a causé beaucoup de joie' sont tout à fait normales, mêmes si certains locuteurs les trouvent stylistiquement maladroites : Vljubivšis'v ètu devušku, Ivan poverg menja v otčajanie et Svoim roždeniem ètot rebënok prinës mne mno-go radosti, cf. (3)a. Cela montre clairement que les contraintes de combinatoire pour les sémantèmes en cause sont propres à des langues particulières. 
Montréal, ou pour un fait qui n’est pas un événement, comme être incapable de réussir un examen, les expressions avec 'causer1' sont possibles :

c. Jean a causé mon désespoir en quittant Montréal / par son incapacité à réussir l'examen.

La contrainte pragmatique stipule que ' $\mathrm{X}$ ' n'est pas un instrument manipulé par quelqu'un dans une actionII.1 concomitante (à l'événement décrit par 'causer1') ; elle est nécessaire pour rendre compte de la phrase (19d) reprise ici sous (27b) :

(27) a. Zoé a causé de grands dégâts à la véranda en la frappant avec un marteau.

b. Le marteau a causé de grands dégâts à la véranda.

Si Zoé a frappé la véranda avec un marteau, on ne peut pas dire (27)b ; cette phrase est bannie par la contrainte pragmatique, parce que le marteau est alors l'instrument manipulé par Zoé (dans une actionII.1 volontaire). Par contre, la phrase (27)b est parfaite, si Zoé a fait tomber le marteau sur la véranda par inadvertance. Ainsi, la structure sémantique suivante est bien formée et peut être appliquée à n'importe quelle situation sous-jacente aux phrases (27), y compris celle où le marteau est un instrument dans la main de Zoé :

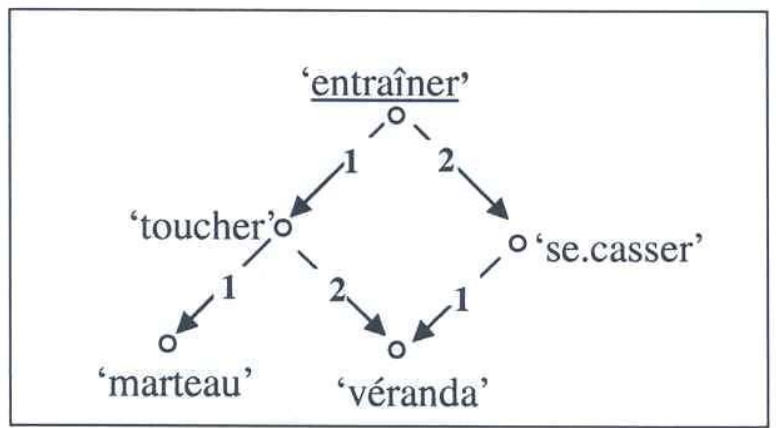

Figure 6 : Une SSém non réductible

Cette SSém peut être réalisée par une phrase comme Le martean a touché la véranda et (de ce fait) elle s'est cassée. Cependant, on ne peut pas réduire cette SSém en appliquant la règle de décomposition de 'causer1' dans une situation où le marteau est un instrument dans la main de quelqu'un. La SSém résultante serait mal appropriée à la situation, et les phrases (grammaticales) qui la réalisent sont inacceptables, toujours vis-à-vis de cette situation.

Notons, comme nous l'a fait remarqué Ju. Apresjan, que si ' $\mathrm{X}$ ' est un moyen (plutôt qu'un instrument) utilisé par quelqu'un dans une actionII.1 concomittante, ' $\mathrm{X}$ ' peut bien être le premier argument de 'causer1' :

(28) a. Le poison utilisé par Zoé a causé la mort de la grenouille.

b. La balle a tué la grenowille.

c. 'Ce nouveau savon a bien nettoyé la baignoire. 
La cooccurrence avec 'causer1' permet ainsi de distinguer entre 'instrument' et 'moyen'.

Comme nous l'avons déjà dit, la contrainte pragmatique est de nature différente par rapport aux contraintes sémantiques: elle porte sur la correspondance entre la structure conceptuelle (grosso modo une représentation du monde) et la SSém et sert à bloquer des configurations sémantiques qui mèneraient à des phrases inappropriées. Elle n'est pas en général vérifiable sur la SSém comme telle : en effet, le sémantème 'instrument' ${ }^{15}$ peut ne pas être présent dans la SSém, bien qu'il s'agisse d'une situation où la contrainte pragmatique s'applique - c'est-à-dire quand ' $\mathrm{X}$ ' est un instrument de quelqu'un même si ce n'est pas explicitement indiqué dans la SSém. La contrainte pragmatique présuppose le recours à un mécanisme capable d'évaluer (la représentation conceptuelle de) la situation décrite et de décider si ' $\mathrm{X}$ ' est ou non un instrument manipulé par quelqu'un dans cette situation.

Pour clore la discussion sur les contraintes sémantiques et pragmatiques de la décomposition de 'causer1', nous souhaitons répondre à la question d'ordre général suivante : pourquoi associer ces contraintes au sémantème 'causer1' plutôt qu'à la lexie correspondante CAUSER1 ? Cette question peut être reformulée de façon encore plus générale :

Faut-il avoir des règles de bonne formation pour la SSém, dont des contraintes sur la COMBINATOIRE SÉMANTIQUE, ou bien faut-il plutôt autoriser n'importe quelles configurations sémantiques et bloquer la réalisation de certaines d'entre elles (celles qui conduisent à des phrases inacceptables) avec des contraintes sur la COMBINATOIRE LEXICALE?

Nous pensons que les contraintes précédentes doivent être formulées, dans la mesure du possible, au niveau sémantique. Voici nos raisons, d'ordre logique et d'ordre linguistique.

D'abord deux considérations d'ordre logique :

- En travaillant avec un langage formel, on préfère contraindre au maximum les expressions possibles dans ce langage ; or les SSém sont des expressions d'un langage formel et nous devons formuler des règles de bonne formation les plus développées et les plus contraignantes possibles.

- Du point de vue de la génération de textes, il est préférable de bloquer le plus tôt possible (et donc au niveau du sémantème plutôt que celui de la lexie) les expressions qui n'auront pas de réalisation linguistique acceptable.

À cela s'ajoutent une considération d'ordre linguistique :

- Tous les verbes causatifs du français partagent certaines propriétés de combinatoire sémantique. En attribuant ces propriétés aux sémantèmes de causation et en considérant que ces sémantèmes apparaissent dans la décomposition de tous ces

15 Le sémantème 'instrument' est un prédicat à quatre arguments: 'X est l'instrument de $\mathrm{Y}$ dans l'actionII.1 Z de Y sur W'. Remarquons que 'instrument' est approximativement équivalent à 'utiliser' : le 'X' que 'Y' utilise est soit un instrument (s'il reste avec 'Y'), soit un moyen (s'il se dissipe sur 'W'; Félix a utilisé du savon de Marseille pour nettoyer sa voiture). 
verbes, on assure automatiquement l'héritage de ces propriétés. C'est le fait d'exprimer ces contraintes comme des conditions portant sur le sémantème 'causer1' plutôt que sur la lexie CAUSER1 qui permet d'assurer un tel héritage. Le fait même que des lexies différentes partagent certaines propriétés devient un critère pour essayer de rattacher ces propriétés à un élément sémantique partagé plutôt qu'à chaque lexie séparément.

Reste que certaines propriétés des lexies ne peuvent pas être naturellement exprimées comme des contraintes sur les sémantèmes. Une lexie peut avoir des conditions de combinatoire qui bloquent/ permettent certaines expressions au-delà des contraintes sémantiques opérant au niveau des sémantèmes. Ainsi, par exemple, la configuration sémantique 'une valise en tombant a blessé Zoé' ne peut pas être transformée en une configuration sémantique telle que *'la chute d'une valise a blessé Zoé' : cela est interdit par la contrainte 2. Cependant, ce sens ne doit pas être proscrit car il peut être lexicalisé par le verbe BLESSER au passif: Zoé a été blessée par la chute d'une valise. On voit ainsi que nos contraintes sur la combinatoire du sémantème 'causer1' ne sont pas tout à fait exactes.

Une dernière précision s'impose ici au sujet du schéma de la Fig. 5 (et par ricochet des règles de décomposition du sémantème 'causer1' de la Fig. 2). Elle concerne l'instanciation des variables ' $\mathrm{Y}$ ' et ' $\alpha$ '.

- 'Y' représente le fait entraîné qui caractérise ' $\alpha$ ', son premier argument ; or, ce fait doit être compris cum grano salis : cela peut être l'absence de fait (c'est-à-dire une négation) ou une phase particulière de fait (début, continuation, ou fin). Ainsi, nos règles de décomposition doivent couvrir la SSém d'expressions comme Le poison a causé l'arrêt de la respiration (où 'Y' = 'arrêt de respiration' plutôt que 'respiration'), etc. La négation et les sens phasiques sont donc transparents pour l'application de la règle. En d'autres termes, 'Y', dans nos règles, vaut éventuellement pour une chaîne de sens comprenant un fait (' $\mathrm{Y}$ ' à proprement parler) et un sens phasique ou la négation.

- ' $\alpha$ ' représente l'entité qui subit l'action $\mathbf{I} .1$ entraînant la causation; mais dans le cas où le patient est une personne (ou un animal), ' $\alpha$ ' peut être une partie du corps de cette personne : La valise a blessé (= a causé une blessure à) Zoé en lui tombant sur le pied. En d'autres termes, ' $\alpha$ ' vaut éventuellement lui aussi pour une chaîne de sens.

Nous nous sommes permis de passer outre ces complications dans notre description sémantique de 'causer1'.

Nous terminerons notre discussion en montrant comment la décomposition de 'causer1' permet de contrôler la bonne utilisation de ce sémantème pour la description langagière d'une situation de causation donnée.

\section{Exemple 1}

Considérons une situation où une grenouille est tuée en touchant un fil électrique sous tension. On peut alors dire (24)a, mais pas(29)b :

(29) a. La décharge électrique a causé la mort de la grenonille.

b. " Le fil électrique a causé la mort de la grenonille.

Le caractère inapproprié de (29)b dans cette situation est bien pris en charge par la décomposition de 'causer1' : dans cette situation, le fil électrique n'a pas agiI.1 sur la grenouille (on ne peut pas dire ${ }^{\#}$ Le fil électrique a agi sur la grenouille à cause de la 
contrainte dite du dernier «chaînon » sur l'usage du sémantème 'agirI.1', formulée p. 258). Par contre, si le fil électrique est tombé sur la grenouille et l'a coupée en deux, le fil a agiI.1 sur la grenouille et (29)b devient appropriée.

\section{Exemple 2}

\# Un bélicoptère américain a causé la mort de trois soldats britanniques.

Cette phrase n'est pas acceptable si l'hélicoptère a largué une bombe sur les soldats ou s'est écrasé avec les soldats à son bord, parce qu'alors l'hélicoptère n'a pas agiI.1 sur eux (la même contrainte sur 'agirI.1' que ci-dessus). Elle n'est acceptable que si l'hélicoptère a agiI.1 sur les soldats, par exemple, en leur tombant dessus. Encore une fois, pour déterminer la bonne formation de la SSém correspondante, il faut consulter une représentation de la situation dont il est question.

\section{Exemple 3}

(31) a. La balle (de la carabine) a causé la mort du loup.

b. "La carabine a causé la mort du loup.

Dans une situation où Zoé a tué un loup en lui tirant une balle de carabine dans la tête, la phrase (31)b n'est pas appropriée, puisque la carabine n'agiI.1 pas sur le loup (toujours la même contrainte sur 'agirI.1'). Dans une autre situation - où Zoé tue le loup en lui assénant un coup de crosse sur la tête - (31)b n’est toujours pas appropriée, car la carabine, bien qu'agissantI.1 maintenant sur le loup, est un instrument entre les mains de Zoé. Mais si la carabine tombe par hasard sur le loup et le tue, (31)b est parfaitement acceptable. Par contre, comme la balle agitI.1 sur le loup et n'est pas un instrument de Zoé, (31)a est normale.

Tout ce que nous venons de dire au sujet de 'causer1' tient pour tous les verbes qui incluent 'causer1' comme composante dominante de leur sens. Ainsi, un verbe comme IRRITER (pris dans l'acception 'causer1 l'irritation'), TUER (= 'causer1 la mort') ou CASSER ( $\sim$ 'causer1 le morcellement') affiche les mêmes propriétés que CAUSER1. Plus précisément, un verbe français avec la composante dominante 'causer1' a les trois propriétés suivantes :

- Il possède au moins trois actants sémantiques : la Cause, le Patient (celui qui subit l'effet de causation ${ }^{16}$ ) et l'Elaboration de la cause ; $c$. :

(32) a. Les voitures irritent Zoé par leur va-et-vient incessant.

b. Le va-et-vient incessant des voitures irrite Zoé par son bruit assourdissant.

c. Le bruit assourdissant du va-et-vient incessant des voitures irrite Zoé par son impact sur ses nerfs auditifs.

${ }^{16}$ Le sens d'effet est nécessairement inclus dans le sens du verbe : l'irritation pour IRRITER, la mort pour TUER, etc. 
- Il hérite du sémantème 'agirI.1', qui est à l'intérieur de 'causer1', de sorte que dans la situation où une grenouille est électrocutée par le contact avec un fil électrique, on peut dire (33)a, mais pas(33)b, puisque le fil n'a pas agiI.1 sur la grenouille :

(33) a. La décharge a tué la grenowille.

b. " Le fil électrique a tué la grenouille.

- Il hérite les conditions d'application du sémantème 'causer1'; par exemple, on ne peut pas dire (34)b si Zoé a frappé la véranda avec un marteau (mais on peut le dire si le marteau est tombé par inadvertance sur la véranda):

(34) a. Zoé a cassé la véranda en la frappant avec un marteau.

b. ${ }^{~}$ Le marteau a cassé la véranda.

Nous pouvons passer maintenant au sémantème 'causer2'.

\section{Le sémantème 'causer2' (= 'être le causateur')}

Le sémantème 'causer2' correspond à la causation agentive (= volontaire) et téléologique (= ayant un but). Alors que 'causer1' repose sur 'agirI.1', 'causer2' repose sur 'agirII.1'. Voici les quatre sens 'agir' considérés dans cette étude (les deux premiers ont été présentés dans la section précédente) :
'agirI.1' $[$ sur $\mathrm{N}] \approx$ 'contact'
'agirI.2' $[\operatorname{sur} \mathrm{N}] \approx$ 'contact' + 'effet'
La gravitation agit sur les objets.
'agirII.1' [sur N] $\approx$ 'action téléologique transitive' Jean agit sur Zoé: par exemple, il la caresse.
'agirII.2' $\approx$ 'action téléologique intransitive' Jean agit : il danse/court/tourne sa tête.

Les paires de sémantèmes 'agirl.1/2' et 'agirII.1/2' s'opposent comme « exprimant un état des choses qui a lieu dans le monde» « exprimant une action volontaire, effectuée par un agent doté de raison ». Les sémantèmes 'agirI.1', 'agirII.1' et 'agirII.2' sont des primitives sémantiques, le deuxième correspondant à 'somebody does something to something/somebody' = 'quelqu'un fait quelque chose à quelqu'un/à quelque chose' de A. Wierzbicka (1996: 50-51, 122-123), et le troisième, à 'somebody does something' $=$ 'quelqu'un fait quelque chose' de A. Bogusławski. Dans Bogusławski 1991 : 46ssv, il est démontré qu'aucun des deux sémantèmes 'agirII.1' et 'agirII.2' ne peut être réduit à l'autre (bien que 'agirII.1' implique 'agirII.2)'. De même que le sémantème 'agirI.1', le sémantème 'agirII.1' = 'actionII.1' semble ne pas avoir une expression lexicale standard en français; le "vrai » verbe AGIRII.1 n'admet pas bien l'expression de son deuxième actant: une phrase comme Zoé a agi sur la grenouille est limite. L'utilisation du verbe AGIRII.1 dans le sens que nous recherchons est néanmoins courante sans l'expression de son deuxième actant : Il a agi énergiquement, Le gowvernement a agi pour enrayer la crise, Essaye d'agir auprès du directeur! Le sémantème 'agirII.1' est très important, et nous nous permettrons de l'utiliser dans les décompositions sémantiques avec un deuxième actant. Comme le présent article ne s'occupe pas de 'agir', cette brève présentation suffira. 
Sylvain Kahane \& Igor Mel'̌uk

Le sémantème 'causer2' a quatre arguments :

[2] 'X cause2 Y par Z(X) (avec W)'

La phrase suivante illustre l'usage du verbe correspondant :

(35) Zoé [= 'X'] a causé la mort [= 'Y'] de la grenouille avec une fourchette [= 'W'] en la lui enfonçant [ = 'Z'] dans l'xil.

L'argument ' $\mathrm{Y}$ ' peut être désigné par la lexie EFFET (de ' $Z$ '), l'argument ' $Z$ ' (l'actionII.1 de 'X') par CAUSE'17 (de 'Y') et l'argument 'W' par INSTRUMENT (de ' $\mathrm{X}$ '). Ces noms peuvent être utilisés dans des paraphrases (comme en (36)b-d). L'argument ' $\mathrm{X}$ ' de 'causer2' n'a pas de nom standard en français, et nous utiliserons le terme technique de Causateur; dans des paraphrases, on peut faire apparaitre, par exemple, RESPONSABLE, AUTEUR, etc. :

(36) a. Zoé [ $[$ ' $X ']$ est responsable de la mort de la grenouille.

b. La mort [ = 'Y'] de la grenouille est l'effet de l'action de Zoé.

c. La fourchette [= 'W'] est l'instrument de Zoé (utilisé pour causer la mort de la grenouille).

d. Le fait d'enfoncer la fourchette dans l'œil de la grenouille [= 'Z'] est la cause de la mort de la grenouille.

L'argument ' $\mathrm{X}$ ' réfère à une personne ou assimilable (animal supérieur, science, machine "intelligente ", personnification - maladie, catastrophe, ...) ; l'argument 'W' est une entité ; les arguments ' $\mathrm{Y}$ ' et ' $\mathrm{Z}$ ' sont nécessairement des faits.

L'argument 'W', l'instrument de 'X' dans l'actionII.1 'Z', est optionnel : 'X' peut agirII.1 sans utiliser d'instrument, notamment si cette actionII.1 est psychologique (Zoé a fâché Félix en le traitant de cornichon).

L'argument ' $Z$ ' est une actionII.1 de 'X'. Contrairement à l'argument ' $Z$ ' de 'causer1', il ne peut pas devenir le premier argument de 'causer2'; il n'est pas une Élaboration de la cause, mais la Cause elle-même. Par conséquent, à la différence de 'causer1', le sémantème 'causer2' n'a qu'une seule «version» de sa décomposition quadri-actancielle -, où le nœud sémantique occupé par l'actionII.1 'Z' est toujours présent. (Cependant, 'Z' ne correspond pas à un actant syntaxique obligatoire et peut bien ne pas être réalisé en surface.)

Le contenu sémantique de 'causer2' peut être représenté en fonction de 'causer1' par la décomposition [3] :

${ }^{17}$ Remarquons que si l'argument ' $Z$ ' est le plus souvent réalisé par un tour gérondif $e n$ $V$-ant (cf. (i)a), un tour gérondif quelconque ne réalise pas forcément l'argument ' $\mathrm{Z}$ ' : cf. (i)b, c où ce tour exprime une circonstance plutôt que la cause de la mort.

(i) a. Zoé l'a tué avec un couteau de cuisine en lui tranchant la gorge.

b. Zoé l'a tué avec un couteau de cuisine en l'attaquant par derrière.

c. Zoé a fait tomber le vase avec son coude en rentrant dans la pièce. 
[3] 'X fait l'actionII.1 Z sur $\alpha$ (en appliquant un instrument W à $\alpha$ ) et Z cause1 $Y$ de $\alpha$ '.

La décomposition du sémantème 'causer2' se présente comme une conjonction logique de deux composantes sémantiques: $\mathbf{A}={ }^{\prime} \mathrm{X}$ fait l'actionII.1 Z sur $\alpha$ (en appliquant un instrument W à $\alpha$ )' et $\mathbf{B}=$ 'l'actionII.1 Z de X sur $\alpha$ (au moyen de W) cause1 Y de $\alpha$ '. L'organisation conjonctive du sens 'causer2' est justifiée par le test de la négation correspondant à la loi de DeMorgan :

$$
\neg(\mathbf{A} \wedge \mathbf{B}) \equiv \neg \mathbf{A} \vee \neg \mathbf{B} .
$$

En effet, la phrase (37)a est équivalente à (37)b :

a. Il n'est pas vrai que Zoé a causé la mort de la grenouille.

b. Il n'est pas vrai que Zoé a agi sur la grenouille ou il n'est pas vrai que, bien que Zoé ait agi sur la grenouille, cette action a causé la mort de la grenouille.

Les verbes français qui incluent le sémantème 'causer2' comme composante principale de leur sens, comme TUER1 $\left(\approx\right.$ 'causer2 la mort') ${ }^{18}$, préservent cette propriété. Ainsi, la phrase (38)a peut être glosée par (38)b :

(38) a. Zoé n'a pas tué la grenouille.

b. Zoé n'a pas agi sur la grenouille ou, bien que Zoé ait agi sur la grenouille, cette action n'a pas causé la mort de la grenouille.

Notons le contraste avec la décomposition de 'causer1' (pour lequel nous n’avons pas utilisé la conjonction) :

(39) a. La balle n'a pas tué (= TUER2, qui comprend 'causer1') la grenonille.

b. Bien que la balle ait agi sur la grenouille, cette action n'a pas causé la mort de la grenouille.

La phrase (39)a ne peut pas signifier *'La balle n'a pas touché la grenouille'. Cet état de choses s'observe également avec d'autres verbes causatifs :

(40) a. Zoé ne s'est pas sali la robe avec de la boue. $\equiv$

Il n'est pas vrai que Zoé a mis sa robe en contact avec la boue, ou il n'est pas vrai que, même si Zoé a mis sa robe en contact avec la boue, la boue a laissé des traces sur la robe.

b. La boue n'a pas sali la robe de Zoé. $\equiv$

Bien que la boue soit entrée en contact avec la robe, elle n'y a pas laissé de traces.

\footnotetext{
18 Nous reviendrons (dans la section 4) sur le fait que l'acception première de TUER (Jean a tué la grenonille), que nous notons TUER1, est basée sur 'causer2', bien que TUER possède une autre acception (La décharge a tué la grenouille), que nous notons TUER2 et qui est basée sur 'causer1'.
} 


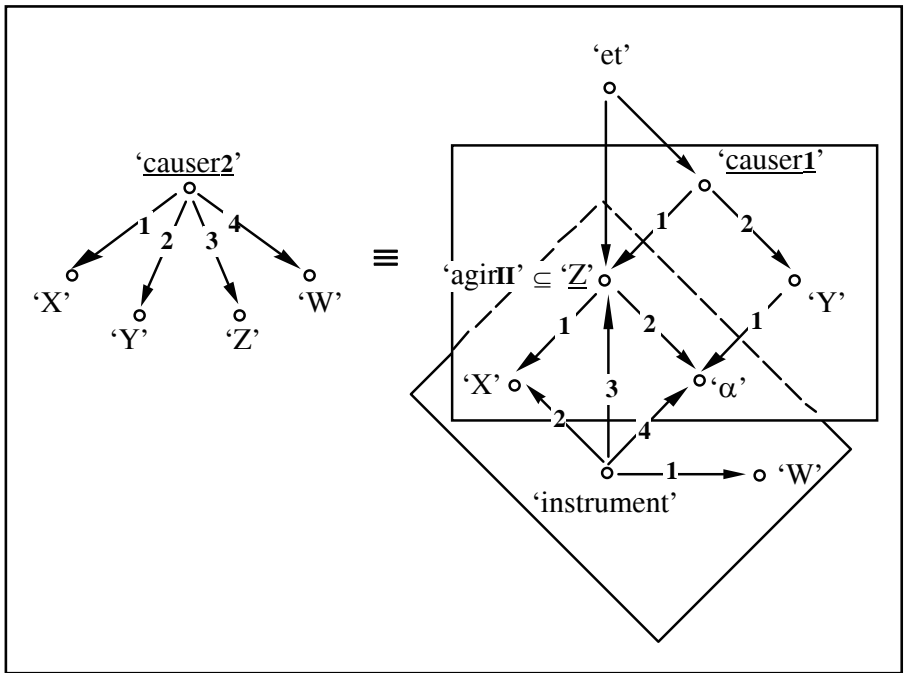

Figure 7 : Décomposition du sémantème 'causer2'

Nous donnons Fig. 7 une décomposition du sémantème 'causer2'; les deux rectangles représentent les deux composantes liées par le connecteur logique 'et' $=$ ' $\wedge$ '. Ce 'et' n'a jamais de contribution substantielle dans le contenu propositionnel de la SSém où il apparaît, mais joue un rôle organisationnel important; nous l'utilisons ici pour indiquer que la SSém de 'causer2' possède deux nœuds communicativement dominants au même niveau. Notre choix d'introduire le sémantème 'et', plutôt qu'un autre moyen formel, repose sur le fait qu'il est lexicalisé par un et dans la glose de la SSém (cf. [3]). (Nous ne représentons pas dans la Fig. 7 le fait que ' $Z$ ' et 'W' sont optionnels; notons que lorsqu'aucun sens spécifique ' $Z$ ' n'est pas exprimé, le sens générique 'agirII.1' figure à la place.)

Nous allons illustrer l'utilisation de la règle de la Fig. 7 et la frontière entre 'causer1' et 'causer2' par un exemple :

\section{a. Les paroles de Jean ont causé l'irritation de Zoé (= ont irrité Zoé).}

b. Jean a causé l'irritation de Zoé $\langle=$ a irrité Zoé) par ses paroles.

D'après notre description, la phrase (41)a contient CAUSER1 ou IRRITER2 (basé sur 'causer1') ; elle reste vague sur la question de savoir si Jean a parlé à Zoé ou à une autre personne. Par contre, (41)b est ambiguë : elle contient soit CAUSER1 ou IRRITER2 - dans le cas où Jean a parlé à quelqu'un d'autre que Zoé (et n'a donc pas agitII.1 sur Zoé), soit CAUSER2 ou IRRITER1 - si Jean adressait ses paroles à Zoé. Pourtant, dans l'intuition d'un locuteur, les phrases (41)a et (41)b sont équivalentes, car elles admettent les mêmes interprétations. Notre modélisation reflète ce fait. Pour l'interprétation où Jean parle à un tiers, l'équivalence est assurée par la règle d'équivalence pour 'causer1' bi- et tri-actanciel (Fig. 1) ; dans l'interprétation où Jean parle directement à Zoé, l'équivalence est assurée par la règle de décomposition sémantique de 'causer2' (Fig. 7). Dans ce dernier cas, il existe un petit décalage entre la SSém de (41)a et la partie droite de la règle qui doit se superposer à cette SSém pour que la règle soit appliquée : la 
partie droite de la règle contient le sémantème 'et' (permettant de mettre la causation1 et l'actionII.1 au même plan communicatif) absent de la SSém de (41)a. On doit passer outre ce décalage, qui est imposé par la nature d'un sémantème (ici 'causer2') et qui ne résulte pas d'un choix du locuteur. Notre système formel d'équivalence sémantique (Kahane \& Mel'čuk 1999, Milićević 2003) devra autoriser certains décalages de ce type lors de l'application de règles d'équivalence.

Indiquons encore une difficulté additionnelle. Les deux phrases en (41) peuvent être perçues comme non équivalentes pour une autre raison. Dans les deux cas, Jean a agiII.1 (il a parlé volontairement), mais (41)b semble impliquer de façon plus nette que Jean a agilI.1 dans le but de causer l'irritation de Zoé. Néanmoins, nous pensons que ceci n'est pas dans le sens de 'causer2', mais résulte plutôt d'une implicature associée à la position sujet du nom humain Jean. On peut en effet bloquer cette implicature par un contexte approprié, comme, par exemple, dans Sans s'en rendre compte, Jean a causé l'irritation de Zoépar ses paroles agressives envers le préposé.

Nous allons maintenant passer à des études de cas pour voir comment les sémantèmes 'causer1' et 'causer2' sont utilisés dans la description de verbes français exprimant une causation.

\section{Verbes causatifs et verbes de causation}

Les verbes qui comprennent dans leur sens les sémantèmes 'causer1/2' en position communicativement dominante se divisent en deux classes :

1) Les verbes causatifs, qui comprennent, à côté de 'causer1/2', une configuration sémantique exprimant l'Effet de la causation en question: TUER $\approx$ 'causer1/2 la mort', où la mort est l'Effet; CONSTRUIRE $\approx$ 'causer2 l'existence', où l'existence est l'Effet; REFLÉTER $\approx$ 'causer1 que l'objet se reflète', où la réflexion de l'objet est l'Effet, etc.

2) Les verbes de causation, qui n'incluent pas de spécification de l'Effet: c'est CAUSER et ses parents, comme DÉCLENCHER, ENTRAÎNER (au sens de 'causer', comme dans Le coup à la tête a entraîné la mort), FAIRE [faire qqch à qqn.], FORCER [qqn. à faire qqch], IMPLIQUER, POUSSER [qqn à faire qqch], etc.

\subsection{Verbes causatifs}

Nous allons commencer notre étude de cas par les verbes causatifs, ce qui nous permet de revenir sur la distinction entre 'causer1' et 'causer2'. Nous avons été amenés à tracer cette distinction par l'existence, en français (ainsi que dans d'autres langues), de trois types de verbes transitifs dont le sens a comme composante principale un sémantème de causation ${ }^{19}$ :

19 Soulignons que nous ne considérons pas ici les sens métaphoriques des verbes analysés. Par exemple, nous écartons les acceptions représentées dans des phrases du type (i) Cette décision va massacrer le projet ou (ii) Ces pensées me torturent: ce sont des métaphores des acceptions qui nous intéressent ici. Le verbe MASSACRER qui apparaît en (i) est MASSACRERII, défini en termes de notre 
(a) Le verbes basés uniquement sur 'causer2' : MASSACRER, CONSTRUIRE, GRILLER, ÉCRIRE, ACHETER, $\ldots{ }^{20}$; leur sujet doit désigner une personne ou quelque chose d'assimilable.

- Les verbes basés uniquement sur 'causer1': REFLÉTER, SÉCHER, ENTRAVER, ...; leur sujet ne peut pas désigner une personne ou quelque chose d'assimilable. (Cf. * Sylvain a séché son slip. Le soleil a séché le slip de Sylvain. Sylvain a fait sécher son slip.)

(b) Les verbes, largement majoritaires, qui ont deux acceptions, la première basée sur 'causer2' et la deuxième sur 'causer1': TUER, DÉTRUIRE, IRRITER, BOUCHER, ...

Le contraste entre ces trois types de verbes causatifs peut être illustré par les exemples en (42):

(42) a. Les rebelles ont massacré ['causer2'] les villageois à coups de machette.

* Les coups de machette ont massacré ['causer1'] les villageois.

b. *Jean a reflété ['causer2'] son visage dans le miroir.

Le miroir a refleté ['causer1'] le visage de Jean.

c. Jean a tué ['causer2'] le loup à coups de machette.

Les coups de machette ont tué ['causer1'] le loup.

La nécessité de distinguer deux acceptions pour les verbes du troisième type peut être étayée par les trois types de considérations suivantes.

\section{Considérations sémantiques}

- Les acceptions basées sur 'causer2' impliquent une action téléologique ('actionII.1') par un humain ou une entité assimilable (animal supérieur, collectivité, ...) sur le premier argument de l'Effet, alors que les acceptions basées sur 'causer1' excluent une telle action. Cela est reflété dans la décomposition sémantique de 'causer2' (Fig. 7), par le fait d'avoir un nœud 'agirII.1'.

- Les acceptions basées sur 'causer2' admettent un réfléchi indirect, alors que les acceptions basées sur 'causer1' ne l'admettent pas :

a. Pierre s'est cassé le bras en tombant.

(Pierre est tombé et, comme résultat, le bras de Pierre s'est cassé.)

b. *La montre s'est cassé l'aiguille en tombant.

(La montre est tombée et, comme résultat, l'aiguille de la montre s'est cassée.)

MASSACRERI comme suit: 'X massacreII $Y^{\prime}=' X$ causel que $Y$ n'existe plus [comme si $\mathrm{X}$ massacraitI Y]'.

${ }^{20}$ Les exemples du type Ce feutre écrit bien, Ce feutre écrit rouge, Ce feutre n'écrit pas sur le plastique ne contredisent pas notre classement du verbe ÉCRIRE, car il s'agit ici d'une autre acception de ce verbe signifiant 'avoir la propriété de pouvoir écrire ...'; cf. l'inacceptabilité de *Ce feutre a écrit cette longue lettre. 
En d'autres termes, on peut se causer2 quelque chose, mais rien ne peut se causer1 quelque chose ${ }^{21}$. Cela résulte du fait qu'on peut agirII.1 sur soi-même, mais pas agirI.1 sur soi-même. Cette différence entre 'causer1' et 'causer2' est reflété par la contrainte $1\left({ }^{\prime} X^{\prime} \neq{ }^{\prime} \alpha\right.$ ' $)$ dans la décomposition de 'causer1', qui n'a pas de contrepartie dans celle de 'causer2'.

\section{Considérations lexicales}

- Les acceptions basées sur 'causer2' et celles basées sur 'causer1' se distinguent par leurs dérivés et leurs cooccurrents. Ainsi, le Causateur de DÉTRUIRE1 [basé sur 'causer2'] s'appelle en français un DESTRUCTEUR, alors que la Cause de DÉTRUIRE2 [basé sur 'causer1'] n'a pas de désignation nominale, mais peut par contre être caractérisée par l'adjectif modificateur DESTRUCTEUR. Cf. :

(44) a. Le général Ngomba a détruit la ville de Goma. [...] Le destructeur $\left\{{ }^{*}\right.$ Le général destructeur) sera traduit devant le TPI.

b. Un séisme de magnitude 5 a détruit la ville de Goma. [...] Le séisme destructeur ${ }^{*}$ Le destructeur) restera dans les mémoires de la population décimée.

De façon similaire, on a à bras raccourcis ou de toutes ses forces comme intensificateur de FRAPPER1 [basé sur 'causer2'], mais de plein fonet pour FRAPPER2 [basé sur 'causer1'].

- Les acceptions basées sur 'causer2' et celles basées sur 'causer1' se distinguent également sous l'angle du paraphrasage par des constructions à verbe support :
a. Félix aide Zoé. $\quad$
Félix donne/ apporte son aide à Zoé.
vs Le médicament aide Zoé. -
* Le médicament donne/apporte son aide à Zoé.
b. Les soldats attaquent le pont. $\sim$ Les soldats lancent une attaque contre le pont. vs L'acide attaque le métal. $\sim$ *L'acide lance une attaque contre le métal.
c. L'armée a détruit le pont. $\quad \sim$ L'armée a procédé à la destruction l*a causé la destruction) du pont.

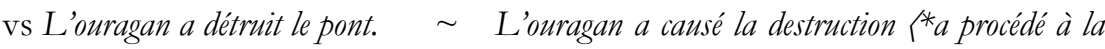 destruction) du pont.
d. Zoé a frappé Félix au front. $\quad \sim$ Zoé a donné à Félix un coup au front.
vs La balle a frappé Félix au front. * * La balle a donné à Félix un coup au front.

\footnotetext{
${ }^{21}$ Attention à ne pas confondre ces exemples avec les paires, assez nombreuses en français, du type casser - se casser (Pierre casse la branche $\sim$ La branche se casse) ou énerver - s'énerver (Pierre énerve Marie $\sim$ Marie s'énerve). Nous estimons que les expressions se casser ou s'énerver ne constituent pas des formes des verbes CASSER ou ÉNERVER. Il s'agit d'un autre lexème - SE CASSER et S'ÉNERVER, dont CASSER et ÉNERVER sont grosso modo des causatifs: 'se casser' $\approx$ 'devenir cassé', alors que 'casser $\mathrm{Y}$ ' $\approx$ 'causer que $\mathrm{Y}$ se casse'; 's'énerver' $\approx$ 'devenir énervé', alors que 'énerver $\mathrm{Y}$ ' $\approx$ 'causer que $\mathrm{Y}$ s'énerve'.
} 


\section{Considérations syntaxiques}

- Les verbes dont le sens est basé sur 'causer2' admettent l'expression de leur actant ' $\mathrm{Z}$ ' (= la Cause de 'Y') par le tour gérondif (cf. (46)a), alors que ceux dont le sens est basé sur 'causer1' ne l'admettent pas (cf. (46)b) :

(46) a. Zoé m'irrite par le bruit qu'elle fait/ en faisant du bruit.

b. Les voitures m'irritent par le bruit qu'elles font/*en faisant du bruit.

- Les deux acceptions ne peuvent pas être coordonnées, cf. 0a, le test de Green-Apresjan; mais la coordination est possible si dans les deux cas il s'agit d'acceptions basées sur 'causer1' :

(47) a. * Les balles et Jean avec son couteau ont tué tous les loups.

b. La forte chaleur et Zoé par son comportement irresponsable m'ont mis hors de moi.

- L'acception agentive est, en règle générale, beaucoup plus fréquente et s'utilise de façon beaucoup moins contrainte que l'acception non agentive (ce phénomène a été noté dans l'article Bogusławski 1991: 42-43, qui défend vigoureusement la distinction entre les deux acceptions des verbes transitifs ${ }^{22}$ ).

Ce dernier point nous incite non seulement à distinguer deux acceptions pour les verbes causatifs mais en plus à considérer que l'acception agentive est le lexème de base du vocable, donc primaire dans un certain sens. Rappelons en effet que c'est l'acception agentive, c'est-à-dire V1, qui :

(a) est caractéristique pour la plupart des verbes transitifs,

(b) peut s'utiliser dans tous les temps grammaticaux, de façon factuelle ou générique, etc. - sans restrictions particulières,

(c) admet un réfléchi indirect (Jean s'est coupé la main),

(d) a des paraphrases verbo-nominales (du type attaquer = lancer une attaque),

(e) a des dérivés actantiels (attaquer: les attaquants, acheter : achat, ...),

(f) accepte le tour gérondif au sens instrumental (Jean m'irrite en produisant ce bruit), etc.

L'acception non agentive, c'est-à-dire V2, n'a pas ces propriétés, ce qui justifie amplement notre choix. D'ailleurs, dans tous les dictionnaires que nous connaissons, et non seulement du français mais de beaucoup d'autres langues, l'acception agentive est toujours sélectionnée comme primaire - pour la très simple raison pratique que

\footnotetext{
22 Bogusławski indique encore un fait linguistique pertinent pour notre discussion : certaines langues permettent les acceptions non agentives plus facilement que les autres. Ainsi, (i) ne peut pas être traduit par un mot-à-mot en polonais ou en russe, car le verbe correspondant dans ces langues n'a pas d'acception non agentive :

(i) The strikes have closed many factories; 'Les grèves ont fermé beaucoup d'usines'.

(ii) Pol. ?? Strajki zamknety wiele fabryk; russe * Zabastovki ostanovili mnogo zavodov.

L'étude de Bogusławski est continuée dans Duraj-Nowosielska 2001, où l'auteur examine d'autres considérations en faveur de la distinction "verbes d'action = causation agentive » vs. " verbes d'événement $=$ causation non agentive $»$.
} 
l'acception non agentive n'existe pas dans beaucoup de cas. En d'autres mots, l'acception V2 - là ou elle existe - est secondaire, "dérivée », et elle est décrite comme une métaphore de V1: 'V2 - comme si c'était V1'. Une telle description rend l'acception V2 formellement secondaire (plus complexe).

Pour illustrer notre propos, nous présenterons ci-dessous les décompositions sémantiques du sens de deux acceptions d'un verbe causatif du français : TUER. D'une part, c'est un très bon représentant des verbes causatifs en général ; d'autre part, il a été profondément étudié dans la littérature linguistique, de sorte que nous pouvons nous baser sur la discussion et les propositions trouvées dans Wierzbicka 1980 : 159ssv.

Deux lexèmes (= acceptions) de TUER seront présentées :

TUER1, comme dans Zoé a tué le loup d'une balle à la tête $\langle$ en lui tirant une balle à la tête $\rangle$; et

TUER2, comme dans La balle 〈Le virus〉 a tué le loup.

Voici la décomposition de 'tuer1' :

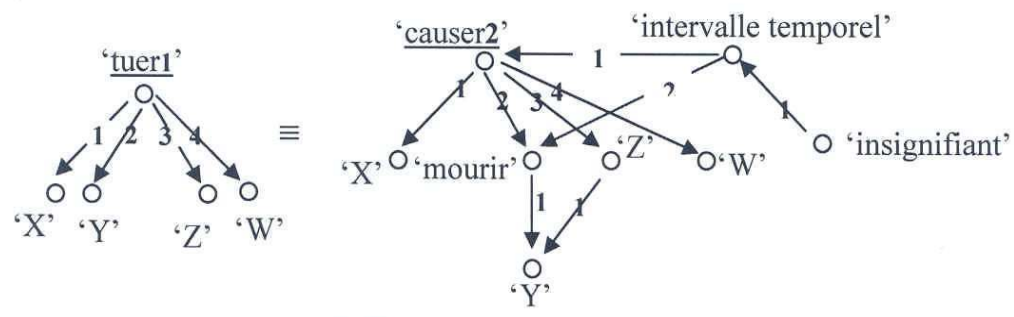

Cette décomposition équivaut à une définition lexicographique verbale suivante :

'X tue1 Y en Z-ant X avec W' $\equiv \quad$ X cause2 que Y meurt en Z-ant X avec W, l'intervalle temporel entre $Z$ et la mort de $\mathrm{X}$ étant insignifiant'

On rend ainsi compte des contrastes suivants :

(48) a. Staline a causé la mort de plusieurs millions de ses concitoyens.

vs ?? Staline a tué plusieurs millions de ses concitoyens.

b. Zoé a tué la grenouille d'un coup de couteau.

vs ??ZZoé a causé la mort de la grenouille d'un coup de couteau. 
Voici, maintenant, notre décomposition pour TUER2 :

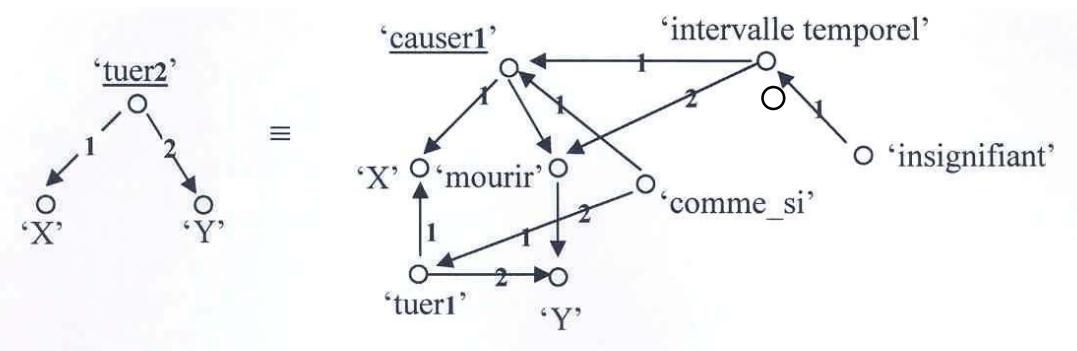

De façon verbale :

'X tue2 $\mathrm{Y}$ ' $\equiv$ 'X cause1 que $\mathrm{Y}$ meurt, l'intervalle temporel entre l'actionI.2 X ou de $\mathrm{X}$ et la mort de $\mathrm{Y}$ étant insignifiant - comme si X tuait1 Y'

Les acceptions non agentives $\mathrm{V} \mathbf{2}$ de verbes agentifs V1 seront toujours basées sur 'causer1'. Pourtant, contrairement à CAUSER1, les verbes V2 n'acceptent pas de sujets exprimant un fait :

(49) a. L'arrivée de Zoé a causé1 la mort de la grenouille.

b. ${ }^{*}$ L'arrivée de Zoé a tue 2 la grenonille.

Nous pensons que le fait que TUER2 est plus restrictif que causer la mort vient du fait que TUER $\mathbf{2}$ est une acception métaphorique de TUER $\mathbf{1}$ et que par conséquent le premier argument de 'tuer2' doit être suffisamment assimilable à un être humain. Il doit donc s'agir soit d'une entité, soit d'un phénomène (voir Annexe), les phénomènes, comme la tornade, l'explosion, le froid, etc., étant assez facilement "personnifiés » :

(50) a. Le marteau (en tombant) a tué la grenouille.

b. Le tsunami a tué plus de cent mille personnes en Indonésie.

Nous rendons compte du caractère métaphorique de TUER 2 et de la contrainte qui en découle par la composante 'comme si X tuait1 Y'. Cette modélisation est loin d'être entièrement satisfaisante. Nous devons en particulier considérer que cette composante de sens est suffisamment faible pour permettre à X d'être non humain et donc de ne pas réellement agirII.2, tout en étant suffisamment forte pour bloquer des $\mathrm{X}$ qui ne pourraient pas être assimilés à des humains.

Si TUER 2 est plus restrictif que causer l la mort, il hérite par contre de toutes les contraintes qui pesaient sur l'emploi de 'causer1', en particulier l'impossibilité d'avoir comme Cause un instrument utilisé dans une actionII.2 concomitante :

* Le marteau (que Zoé tenait fermement dans la main) a tué la grenouille.

Nous terminons ici notre description des sens de verbes causatifs et passons aux verbes de causation. 


\subsection{Verbes de causation}

Nous allons maintenant présenter la décomposition du sens d'un verbe de causation : le verbe anglais [to] HAVE, pris dans la construction causative du type $\mathrm{He}$ had her bring him the book. Nous avons choisi ce verbe car il est sémantiquement analysé dans Wierzbicka 1998 ; nous reprenons sa décomposition et la traduisons dans notre langage sémantique - structure sémantique en forme de réseau. Il nous semble intéressant de choisir cet exemple, car il illustre une nuance subtile du sens de causation qui n'est pas représentée en français, mais que nos deux sémantèmes de causation - 'causer1' et 'causer2' - permettent d'expliciter. Nous supposons bien évidemment qu'en anglais il faut distinguer deux sémantèmes 'cause1' et 'cause2', équivalents aux sémantèmes français 'causer1' et 'causer2'. Les contraintes sur la combinatoire sont naturellement différentes en anglais et en français, mais les sémantèmes eux-mêmes semblent être dans une correspondance bi-univoque.

Voilà la décomposition du verbe de causation [to] HAVEII par Wierzbicka, comme on le voit dans la phrase (52), citée par elle :

She had the girls clean bis bicycle.

(53) Person $\mathrm{X}$ had person $\mathrm{Y}$ do $\mathrm{Z}=$

(a) $\mathrm{X}$ wanted $\mathrm{Z}$ to happen (to $\mathrm{W}$ )

(b) because of this $\mathrm{X}$ wanted $\mathrm{Y}$ to do $\mathrm{Z}$ (to $\mathrm{W}$ )

(c) because of this $\mathrm{X}$ said something to somebody

(d) because of this $\mathrm{Y} \operatorname{did} \mathrm{Z}$

(e) $\mathrm{X}$ could think that when $\mathrm{X}$ says something like this (about something like this) Y can't say "I don't want to do this"

Nous allons nous baser sur cette définition pour notre décomposition. Les modifications que nous effectuons résultent de deux choses.

D'une part, nos buts linguistiques et nos principes de description diffèrent quelque peu de ceux de Wierzbicka :

Wierzbicka cherche à découvrir le langage universel de la pensée humaine ; ainsi elle utilise dans ses décompositions des éléments sémantiquement les plus simples possible, c'est-à-dire les plus proches des primitives sémantiques, ce qui l'amène à décomposer chaque sens au maximum et à utiliser un métalangage sémantique très contrôlé. Nous, par contre, avons pour but de construire des dictionnaires formels de langues particulières sans chercher nécessairement des éléments sémantiques universels ; notre principe est de décomposer chaque sens au minimum en suivant le principe du bloc maximum (c'est-à-dire de ne pas décomposer un sens lorsqu'il existe, dans la langue qu'on est en train de décrire, un sémantème qui exprime ce sens). Notre métalangage sémantique, par conséquent, n'est pas aussi limité que celui de Wierzbicka.

D'autre part, quelques points nécessitent à notre avis d'être modifiés : 
- Le verbe 'say' (= 'dire') en (c) ne nous paraît pas un bon choix, car X peut have $Y$ do $Z$ sans que $\mathrm{X}$ dise quoi que ce soit: $\mathrm{X}$ peut par exemple écrire ou exprimer sa volonté par des gestes. Nous remplaçons donc 'say' par 'ask' [somebody to do something] (= 'demander' [à qqn de faire qqch]). Ce sémantème couvre en fait d'un coup les lignes (a) - (c) de la définition de Wierzbicka. En effet, ' $\mathrm{X}$ asks $\mathrm{Y}$ to do Z' présuppose que ' $\mathrm{X}$ ' veut que ' $\mathrm{Z}$ ' ait lieu et que ' $\mathrm{X}$ ' communique sa volonté à ' $\mathrm{Y}$ ' dans le but de l'inciter à faire ' $Z$ '. (C'est le principe du bloc maximal qui nous amène à utiliser le sémantème 'ask' plutôt qu'une décomposition équivalente ; pour l'analyse sémantique de ASK, voir Wierzbicka 1987 : 49-50.) La composante (c) indique qu'il n'est pas nécessaire que $\mathrm{X}$ s'adresse directement à $\mathrm{Y}$ : il peut lui faire connaitre sa volonté par un tiers. Mais 'ask' = 'demander' peut également être utilisé sans qu'on s'adresse directement au destinataire (cf. Je vais demander ça à Jean par l'intermédiaire de ma secrétaire), de sorte que la composante 'said something to somebody' est reflétée. Comme Wierzbicka nous l'a fait remarquer, 'ask Y to do Z' inclut la composante 'Y n'est pas obligé de faire $Z$ '. Or cette composante pourrait entrer en contradiction avec le sens 'have Y do Z'. Nous pensons que ce n'est pas un problème, car la composante en question est faible : elle peut facilement être bloquée par un autre élément de sens. Ainsi un directeur peut bien ask his secretary to do $Z$, même si $Z$ fait partie des obligations professionnelles de la secrétaire.

- Nous avons fait de la composante (d) notre composante communicativement dominante 'cause2'. Cela représente une modification substantielle. D'une part, dans sa formulation de (d), Wierzbicka privilégie une version adverbiale du sémantème 'cause1' - 'because of', tandis que nous préférons la version verbale (qui nous parait plus appropriée pour être utilisée dans des décompositions de sémantèmes verbaux). D'autre part, Wierzbicka ne montre pas explicitement les composantes communicativement dominantes et donc le sens que nous considérons comme communicativement dominant dans 'haveII' - 'cause2' - n'apparaît qu'en (d) ; notre décomposition indique que 'haveII' est grosso modo 'cause2'. (Dans notre approche, le nœud dominant d'une définition doit être sa paraphrase minimale. En raison de l'exigence du bloc maximal, nous devons utiliser 'cause2' plutôt qu'une version décomposée avec 'cause1', qui serait plus proche de la composante (d) de Wierzbicka.) Enfin, Wierzbicka indique que 'Y did Z', alors que dans notre représentation l'idée d'implicativité de 'haveII' est subsumée par l'utilisation de 'cause2', qui lui est implicatif.

- Nous avons modifié l'énoncé de (e) en lui préférant la composante sémantique suivante: 'le fait que $\mathrm{X}$ domine socialement $\mathrm{Y}$ lui fait croire qu'il est impossible que $\mathrm{Y}$ refuse de faire $\mathrm{Z}$ '. Ceci reste assez conforme à la formulation de Wierzbicka, car même si elle ne spécifie pas les rapports sociaux entre X et $\mathrm{Y}$ dans la décomposition de 'haveII', elle insiste sur leur existence dans ses commentaires. Mais surtout, la composante (e) de la décomposition de Wierzbicka correspond chez nous à un présupposé, car elle reste affirmée sous l'interrogation (Did she have the girls clean his bicycle? permet d'inférer qu'elle se trouve par rapport aux filles dans une position sociale qui lui permet de croire qu'elles ne refuseront pas si elle le demande). 
Notre décomposition de HAVEII prend ainsi la forme de la règle suivante :

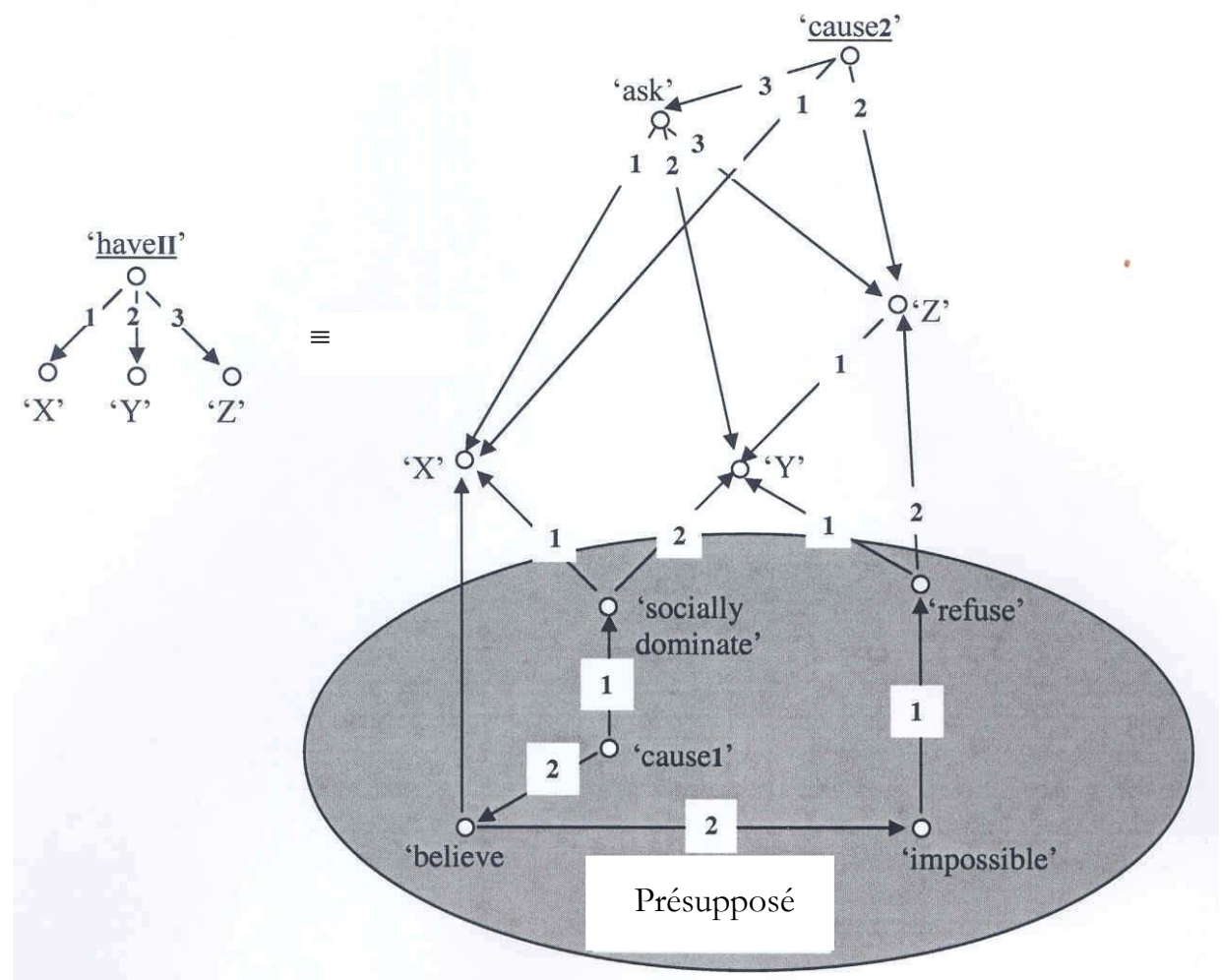

Figure 9 : Décomposition du sémantème anglais 'haveII'

\section{Conclusion}

Nous espérons avoir montré que le sens langagier de causation peut être assez bien décrit en français grâce aux deux sémantèmes proposés - 'causer1' et 'causer2'. On peut croire que ces sémantèmes sont relativement universels et que la distinction entre eux devra être maintenue dans toutes les langues. Bien que nous ne les considérons pas comme des primitives sémantiques, ils en sont très proches et servent à décrire à la fois les autres verbes de causation et les verbes causatifs.

Nous avons montré que le sémantème 'causer1' a une structure actancielle assez spécifique, puisqu'il a deux patrons équivalents d'arguments sémantiques - bi-actanciel et tri-actanciel, avec un actant escamotable (' $\mathrm{Z}(\mathrm{X})$ causer1 $\mathrm{Y}$ ' $\equiv{ }^{\prime} \mathrm{X}$ causer1 Y par Z(X)') et qu'il permet l'« alternance » sémantique entre son premier et son troisième argument par un jeu de chaises musicales: 'X causer1 Y par $\mathrm{Z}(\mathrm{X})^{\prime} \equiv{ }^{\prime} \mathrm{Z}(\mathrm{X})$ causer1 Y par $\mathrm{Z}_{1}(\mathrm{Z}(\mathrm{X}))^{\prime} \equiv \ldots$ (comme, par exemple, Zoé irrite Félix par ses remarques $\equiv$ Les remarques de Zoé irrite Félix par leur méchanceté $\equiv$ La méchanceté des remarques de Zoé irrite Félix ...) 
Notre étude pour le français a mise à jour plusieurs contraintes sur la combinatoire du sémantème 'causer1' (voir Fig. 5). La formulation de telles contraintes permet de jeter les bases des règles de bonne formation des structures sémantiques telles qu'elles sont envisagées dans le cadre de la théorie Sens-Texte. Il serait intéressant de voir à quel point les mêmes contraintes sont valables dans d'autres langues. (Nous avons vu, par exemple, section 2, que les contraintes ne sont pas les mêmes pour les sémantèmes 'agirI.1' en français et 'actI.1' en anglais.) Certaines contraintes sur la combinatoire de 'causer1' (comme sur celle de 'causer2') ne sont pas strictement parlant sémantiques : elles doivent être formulées comme des propriétés de la situation décrite par le prédicat verbal - comme, par exemple, l'impossibilité pour la Cause d'être un instrument de quelqu'un dans une actionII.1 concomitante de ce quelqu'un. De telles contraintes touchent à la façon dont sont élaborées les représentations sémantiques du message à communiquer à partir de la situation extralinguistique. Il s'agit d'un domaine encore inexploré dans le cadre de la théorie Sens-Texte, qui prend normalement la représentation sémantique comme entrée de sa description d'une production langagière.

Notre travail sur la causation en français mériterait d'être approfondi dans plusieurs directions.

- Dans un premier temps, nous devrions poursuivre notre description des verbes causatifs et de causation en l'appliquant à des centaines de verbes. On pourra considérer l'emploi des sémantèmes 'causer1' et 'causer2' dans les définitions lexicographiques des verbes et tester leur comportement dans les paraphrases.

- Il serait intéressant de relier la description de verbes causatifs à la description de sens causatifs rendus par les connecteurs (conjonctions + prépositions) et de les harmoniser: Zoé a renversé Félix Félix est tombé parce que Zoé l'a poussé. Un cas relié est l'expression du lien causal par la seule juxtaposition (accompagnée d'une prosodie appropriée) : Félix est tombé-Zoé l'a poussé.

- Il faut appliquer le même type de description aux constructions verbales causatives du français (avec FAIRE et LAISSER).

\section{Annexe}

Dans les décompositions sémantiques des lexies, un rôle tout particulier revient à quelques sémantèmes très généraux, dont chacun est utilisé dans la définition d'un nombre élevé de sens lexicaux en contrôlant la cooccurrence lexicale libre des lexies ; on les appelle « étiquettes sémantiques » (Polguère 2003). Sans entrer dans les détails concernant les étiquettes sémantiques et leur hiérarchie, nous présenterons ici celles qui sont utilisées dans le présent article.

Pour rendre accessible la notion d'étiquetage sémantique au lecteur, indiquons que, grosso modo, une étiquette sémantique de la lexie L n'est pas autre chose que la spécification du genre prochain de $\mathrm{L}$ dans la définition analytique de L. Quant aux sens qui ne peuvent pas être réellement décomposés, comme les primitives et quasiprimitives sémantiques, l'étiquette sémantique leur est attribuée de l'« extérieur»par une caractérisation ; c'est le cas de 'causer1' que nous déclarons être un 'événement', bien que nous l'ayons décomposé et que son genre prochain soit 'entraîner'. 
Soulignons que les sémantèmes 'fait', 'entité, 'événement', etc. sont des sens des lexies françaises correspondantes, pas des construits philosophiques ou logiques. Leur contenu sémantique n'est donné que par leur cooccurrence lexicale et syntaxique observable : notre seul critère est ce qu'on peut/ne peut pas dire en français.

Les deux étiquettes sémantiques les plus générales sont 'fait' vs 'entité' :

- Un 'fait' est un sémantème 'L' qu'on peut enchâsser sous 'Je sais que ...' et dont on peut affirmer que 'L a lieu'. En d'autres mots, un 'fait' correspond toujours à un prédicat et vice versa. Attention à ne pas confondre notre emploi de 'fait' avec d'autres acceptions du vocable FAIT : ainsi trouve-t-on souvent le nom fait utilisé pour référer à un événement (Ces faits se produisent toujours dans les mêmes conditions) ou à une proposition vraie (Zoé est très belle: c'est un fait).

- Une 'entité' est un sémantème 'L' qu'on ne peut pas enchâsser sous 'Je sais que ...' et dont on ne peut pas affirmer ' $\mathrm{L}$ a lieu'. Une 'entité' peut être un 'objet', une 'substance' ou un 'être (animé)'. Parmi les êtres animés, une place importante revient à 'individu' (= un être humain) et à 'personne', qui couvre 'individu', mais aussi les entités assimilables comme 'ensemble d'individus', 'société, 'établissement', 'État', 'machine (suffisamment) autonome', 'science', idéologie', ...

Sous 'fait' on trouve :

- 'événement', dont on peut dire qu'il 'se produit' et qui se définit par la possibilité d'accepter la caractérisation temporelle: un événement se produit à un moment donné. Parmi les événements, on trouve des 'phénomènes', comme 'exploser', 'tomber', 'tremblement de terre', 'pleuvoir') et les 'actionsI.1,I.2', y compris 'causer1';

- 'actionII.1/2', que X effectue en tant qu'Agent ('écrire', 'tuer', 'couper'), y compris 'causer2';

- 'processus', comme 'agoniser' ou 'se développer';

- 'état', dans lequel 'X peut se trouver'; on distingue plusieurs types d'états :

- 'état physique' ['cassé', 'débout', 'blessé', ...], c'est-à-dire qqch de visible/tangible ;

- 'état physiologique' ['fatigué', 'affamé', 'malade', ...] ;

- 'état psychologique' ['étonné', 'indigné', 'vouloir', 'aimer', ...], etc.;

- 'caractéristique', que 'X peut posséder' :

- 'propriété' ['couleur', 'utilité', 'importance', ...] ;

- 'paramètre' ['dimension', 'poids', 'vitesse', ...].

- 'relation', qui 'relie $\mathrm{X}$ et $\mathrm{Y}$ ' ['X précède $\mathrm{Y}$ ', 'X est un étudiant de $\mathrm{Y}$ ', 'X est la mère de Y']; etc.

\section{Remerciements}

En écrivant cet article, nous avons profité des critiques et des suggestions des personnes suivantes : M. Alonso Ramos, Ju. Apresjan, L. Barque, L. Iordanskaja, J. Milićević et Anna Wierzbicka. Grâce à leurs critiques et suggestions nous avons pu (espérons-le !) améliorer l'exposé de façon significative.

L'article a été écrit lors du séjour d'I. Mel'čuk à Paris en tant que titulaire d'une Chaire Internationale Blaise Pascal, gérée par la Fondation de l'École Normale Supérieure et financée par la Région Ile de France. 
Sylvain Kahane \& Igor Mel'cuk

\section{RÉFÉRENCES}

Anscombre, Jean-Claude, 1984, La représentation de la notion de cause dans la langue. Cabiers de grammaire, $\mathrm{n}^{\circ} 8,3-53$.

Apresjan, Jurij, 1974, Leksičeskaja semantika. Sinonimičeskie sredstva jasylka. Moskva: Nauka.

APRESJAN, Jurij, 1995 [1994], O jazyke tolkovanij i semantičeskix primitivax. In: Ju. Apresjan, Izbrannye trudy, tom II, Integral'noe opisanie jasylka i sistemnaja leksikografija, Moskva: Škola «Jazyki russkoj kul'tury», 466-484.

APresjAn, Jurij, 1998 [1976], Kauzativy ili konversivy ? In: N. Kozinceva \& A. Ogloblin (red.), Tipologija. Grammatika. Semantika (K 65-letiju V.S. Xrakovskogo), Sankt-Peterburg: Nauka, 273-281.

BogusŁawski, Andrzej, 1991, Semantic Primes for Agentive Relations. Lingua Posnaniensis, vol. 32-33, 39-64.

COMrIE, Bernard, 1974, Causatives in Universal Grammar. Philological Society's Transactions, 1-32.

COMrIE, Bernard, 1985, Causative Verb Formation and Other Verb-Deriving Morphology. In: Th. Shopen (ed.), Language Typology and Syntactic Description, Cambridge: Cambridge University Press, 3: 309-348.

Duraj-Nowosielska, Izabela, 2001, O ‘działaniach' i 'wypadkach' - równobrzmiące wielowalentne czasowniki s podmiotami osobowymi (kto) i nieosobowymi (co). Bulletin de la société polonaise de linguistique, fasc. 57, 21-54.

Ehring, Douglas, 1997, Causation and Persistence. A Theory of Causation. New York-Oxford: Oxford University Press.

GIVón, Talmy, 1975, Cause and Control: On Semantics of Interpersonnal Manipulation. In: J. Kimball (ed.), Syntax and Semantics. 4, New York: Academic Press, 59-89.

Goldvarg, Eugenia \& Johnston-LAird, Philip, 2001, Naive Causality: A Mental Model Theory of Causal Meaning and Reasoning. Cognitive Science, 25, 565-610.

Gross, Gaston, 2005, La typologie des relations causatives [inédit; une communication présentée à la Société de linguistique de Paris le 15 janvier 2005].

GuILLET, Alain \& LECLÈRE, Christian, 1981, Restructuration du groupe nominal. In : A. Guillet \& C. Leclère (réd.), Formes syntaxiques et prédicats sémantiques [= Langage, 63], 99-128.

IORDANSKAJA, Lidija, 1999, Description lexicographique des conjonctions du français contemporain. In: Mel'čuk et al., Dictionnaire explicatif et combinatoire du français contemporain. Recherches lexico-sémantiques IV, Montréal: Les Presses de l'Université de Montréal, 43-53.

IORDANSKAJA, Lidija \& ARBATCHEWSKI-JUMARIE, Nadia, 2000, Quatre prépositions causales du français contemporain. Leur sémantisme et cooccurence. Lingvistica Investigationes, 23 : 1, 115-159. 
IORDANSKAJA, Lidija \& MEL'ČUK, Igor, 2001, Conversif ou causatif ? Cabiers de lexicologie, $80: 1$, 105-119.

KAHANE, Sylvain \& MEL'ČUK, Igor, 1999, Synthèse de phrases à extraction en français contemporain (du réseau sémantique à l'arbre syntaxique). TAL, $40: 2,25-85$.

KISTLer, Max, 1999, Causalité et lois de la nature. Paris : J. Vrin.

MeL'ČUK, Igor, 2001, Communicative Organization in Natural Language, Amsterdam/Philadelphia: Benjamins.

MEL'ČUK, Igor, 2004a, Actants in Semantics and Syntax I: Actants in Semantics. Linguistics, 42: 1, 1-66.

MEL'ČUK, Igor, 2004b, Actants in Semantics and Syntax II: Actants in Syntax. Linguistics, 42: 2 , 247-291.

MiLIĆEviĆ, Jasmina, 2003, Modélisation sémantique, syntaxique et lexicale de la paraphrase. Montréal : Université de Montréal. [Thèse de doctorat.]

NAZARENKO, Adeline, 2000, La cause et son expression en français. Ophrys : Gap - Paris.

PaducheVA, Elena, 1997, Verb Categorization and the Format of a Lexicographic Definition (Semantic Types of Causative Relations). In: L. Wanner (ed.), Recent Trends in MeaningText Theory, Amsterdam/Philadelphia : Benjamins, 61-73.

PolguÈre, Alain, 1990, Structuration et mise en jeu procéduraled'un modèle linguistique déclaratif dans un cadre de génération de texte. Montréal : Université de Montréal. [Thèse de doctorat.]

Polguère, Alain, 1992, Remarques sur les réseaux sémantiques Sens-Texte. In : A. Clas (réd.), Le mot, les mots, les bons mots, Montréal : Presses de l’Université de Montréal, 109-148.

Polguère, Alain, 2003, Étiquetage sémantique des lexies dans la base de données DiCo, TAL, $44: 2,39-68$.

Shibatani, Masayoshi, 1973, Semantics of Japanese causativization. Foundations of Language, 9 , 327-373.

Shibatani, Masayoshi (ed.), 1976, The Grammar of Causative Constructions (Syntax and Semantics. O), New York : Academic Press.

Song, Jae Jung, 1996, Casatives and Causation: A Universal-Typological Perspective. London : Longman.

ST-GERMAIN, Jean, 1995, Incidence de la structure sémantique et communicative sur la structure syntaxique profonde des énoncés causatifs du français contemporain. Montréal: Université de Montréal. [Thèse de doctorat.]

TALmy, Leonard, 1988, Force Dynamics in Language and Cognition. Cognitive Science, 12: 49-100.

Tooley, Michael, 1987, Causation. A Realist Approach. Oxford: Clarendon Press.

ToOley, Michael, 1997, Time, Tense, and Causation. Oxford: Clarendon Press.

WierzBickA, Anna, 1980, Lingua Mentalis. Sydney etc.: Academic Press.

WierzBICKA, Anna, 1987, English Speech Act Verbs: A Semantic Dictionary. Sydney: Academic Press. 
Sylvain Kahane \& Igor Mel'cuk

WierzBickA, Anna, 1996, Semantics: Primes and Universals. Oxford: Oxford University Press.

WierzBickA, Anna, 1998, The Semantics of English Causative Constructions in a UniversalTypological Perspective. In: M. Tomasello (ed.), The New Psychology of Language: Cognitive and Functional Approaches to Language Structure, New Jersey: Lawrence Erlbaum, 113-153.

WierzBICKA, Anna, 2004, English Causative Constructions in an Ethnosyntactic Perspective: Focussing on LET. In: N.J. Enfield (ed.), Ethnosyntax, Oxford: Oxford University Press, 162-203.

WolfF, Philip, 2003, Direct Causation in the Linguistic Coding and Individuation of Causal Events. Cognition, 88, 1-48. 